\title{
Alteration of the cutaneous microbiome in psoriasis and potential role in Th17 polarization
}

\author{
Hsin-Wen Chang ${ }^{1}$, Di Yan ${ }^{1,2}$, Rasnik Singh ${ }^{1,4}$, Jared Liu, Xueyan Lu ${ }^{1,5}$, Derya Ucmak ${ }^{1,6}$, Kristina Lee ${ }^{1}$, Ladan Afifi ${ }^{1,3}$, \\ Douglas Fadrosh”, John Leech', Kimberly S. Vasquez', Margaret M. Lowe', Michael D. Rosenblum', \\ Tiffany C. Scharschmidt', Susan V. Lynch ${ }^{7}$ and Wilson Liao ${ }^{1 *}$
}

\begin{abstract}
Background: Psoriasis impacts 1-3\% of the world's population and is characterized by hyper-proliferation of keratinocytes and increased inflammation. At the molecular level, psoriasis is commonly driven by a Th17 response, which serves as a major therapeutic target. Microbiome perturbations have been associated with several immune-mediated diseases such as atopic dermatitis, asthma, and multiple sclerosis. Although a few studies have investigated the association between the skin microbiome and psoriasis, conflicting results have been reported plausibly due to the lack of standardized sampling and profiling protocols, or to inherent microbial variability across human subjects and underpowered studies. To better understand the link between the cutaneous microbiota and psoriasis, we conducted an analysis of skin bacterial communities of 28 psoriasis patients and 26 healthy subjects, sampled at six body sites using a standardized protocol and higher sequencing depth compared to previous studies. Mouse studies were employed to examine dermal microbial-immune interactions of bacterial species identified from our study.

Results: Skin microbiome profiling based on sequencing the $16 \mathrm{~S}$ rRNA V1-V3 variable region revealed significant differences between the psoriasis-associated and healthy skin microbiota. Comparing the overall community structures, psoriasis-associated microbiota displayed higher diversity and more heterogeneity compared to healthy skin bacterial communities. Specific microbial signatures were associated with psoriatic lesional, psoriatic non-lesional, and healthy skin. Specifically, relative enrichment of Staphylococcus aureus was strongly associated with both lesional and non-lesional psoriatic skin. In contrast, Staphylococcus epidermidis and Propionibacterium acnes were underrepresented in psoriatic lesions compared to healthy skin, especially on the arm, gluteal fold, and trunk. Employing a mouse model to further study the impact of cutaneous Staphylcoccus species on the skin T cell differentiation, we found that newborn mice colonized with Staphylococcus aureus demonstrated strong Th17 polarization, whereas mice colonized with Staphylococcus epidermidis or un-colonized controls showed no such response.
\end{abstract}

Conclusion: Our results suggest that microbial communities on psoriatic skin is substantially different from those on healthy skin. The psoriatic skin microbiome has increased diversity and reduced stability compared to the healthy skin microbiome. The loss of community stability and decrease in immunoregulatory bacteria such as Staphylococcus epidermidis and Propionibacterium acnes may lead to higher colonization with pathogens such as Staphylococcus aureus, which could exacerbate cutaneous inflammation along the Th17 axis.

\footnotetext{
* Correspondence: Wilson.Liao@ucsf.edu

${ }^{1}$ Department of Dermatology, University of California, San Francisco, CA

94115, USA

Full list of author information is available at the end of the article
}

(c) The Author(s). 2018 Open Access This article is distributed under the terms of the Creative Commons Attribution 4.0 International License (http://creativecommons.org/licenses/by/4.0/), which permits unrestricted use, distribution, and reproduction in any medium, provided you give appropriate credit to the original author(s) and the source, provide a link to the Creative Commons license, and indicate if changes were made. The Creative Commons Public Domain Dedication waiver (http://creativecommons.org/publicdomain/zero/1.0/) applies to the data made available in this article, unless otherwise stated. 


\section{Background}

Psoriasis is an immune-mediated inflammatory skin disease that impacts $1-3 \%$ of the world's population. The pathogenesis of psoriasis is multifactorial with notable contributions from patient genetics and environmental factors such as lifestyle, diet, and health history [1, 2]. Psoriasis can be mediated by an overactive Th17 response leading to skin inflammation and hyper-proliferation of keratinocytes [3]. In the clinic, blocking components of the Th17 pathway effectively dampens the aberrant immune response in psoriasis patients and controls symptoms, but these treatments do are not curative and disease management effectiveness varies across patients. This highlights the need to further understand the pathogenesis of psoriasis and the factors associated with disease initiation and progression.

The skin is the human body's largest organ which serves not only as a physical protective barrier against environmental insults, but also as a dynamic interface for host dermal-microbial interactions. The microbial community that inhabits the human skin is highly complex and consists of highly diverse microorganisms including bacteria, fungi, viruses, and archaea [4]. Bacteria have been shown to be essential for skin health by restricting pathogen colonization and fine-tuning resident $\mathrm{T}$ cell function $[5,6]$. As a result, perturbations to the skin microbial community have the potential to contribute to altered skin immune function. Indeed, dysbiosis of the skin microbiome has been linked to several inflammatory and autoimmune diseases including atopic dermatitis and vitiligo [7, 8], suggesting the importance of the cutaneous microbiome in the health of the skin.

Interestingly, throat and nasal Streptococcal infection have been shown to trigger initiation and exacerbation of psoriasis $[9,10]$, suggesting a microbial contribution to disease. Moreover, keratinocytes, the most prominent cell type in the epidermis, can trigger innate and adaptive immune responses in psoriasis through interactions with skin bacteria [11]. To date, several studies have sought to characterize the psoriasis-associated skin microbiome and identify bacterial species that might contribute to the pathogenesis of psoriasis [12-16]. However, these studies revealed a lack of consensus on psoriasis-associated microbial signatures plausibly due to the inherent heterogeneity of microbial species that promote immune dysfunction in psoriatic patients and or to different study designs. For example, collecting samples using skin swabs [12, 14] or skin biopsies [13] introduces significant variability since these methods sample different cutaneous anatomical compartments with likely different associated bacteria [17]. Moreover, these studies used different $16 \mathrm{~S}$ rRNA primers amplifying different variable regions of the $16 \mathrm{~S}$ rRNA gene, which may contribute to variance across studies, making cross study comparisons difficult. Therefore, application of a standardized protocol to allow for a better understanding in the relationship between microbiome and disease is critical $[17,18]$.

In this study, we surveyed the skin microbiome from 28 psoriasis patients and 26 healthy subjects using the standardized protocol recommended by the NIH Human Microbiome Project [19-21]. In contrast to some previous studies targeting the V4 region of the $16 \mathrm{~S}$ rRNA gene [13], we profiled the skin microbial community using primers targeting the V1-V3 region, which results in more accurate bacterial identities of the skin microbiome at the genus and species levels compared to the traditional V4 approach [20, 22]. We also used higher sequencing depth to ensure high-quality data. Our data revealed significant alterations in the psoriasis skin microbiome and identified Staphylococcus aureus as a potential contributor to psoriasis pathogenesis.

\section{Results}

\section{Cohort of patients and skin sampling}

The cohort in this study consisted of 28 patients with plaque psoriasis and 26 healthy individuals. To avoid any confounding demographic effects, gender and age composition were matched between the two groups (Table 1). All psoriasis patients were clinically diagnosed with psoriasis at the UCSF Psoriasis and Skin Treatment Center. The psoriasis patients in this study had a mean Psoriasis Area and Severity Index (PASI) of 11.1 representing moderate-to-severe disease. To avoid the variabilities introduced by treatments, we excluded subjects with recent antibiotic treatment and/or other biologic and systemic therapy. In addition, all subjects required to undergo a 2-week wash-out period for topical therapy. Different anatomic sites in the human skin can be categorized into three major groups: dry, moist, and sebaceous. The biogeographical differences across different skin sites provide different environments that support distinct microbial communities [23-26]. In order to gain a comprehensive view of the psoriasis-associated skin microbiome, we sampled the microbiome across six different skin sites: scalp, trunk, arm, leg, axilla, and gluteal fold, which covers all three skin groups (Table 2). Three different "disease states" were sampled for each skin site: healthy skin from healthy subjects (Healthy), unaffected or non-lesional skin from psoriasis patients (PSO_N), and lesional skin from

Table 1 Demographic information of study cohort

\begin{tabular}{llll}
\hline & Healthy subjects & Psoriasis subjects & $p$ value \\
\hline Sample size & 26 & 28 & NA \\
Mean age (years) & $42.3 \pm 14.1$ & $43.6 \pm 15.1$ & 0.75 \\
Gender (\%Female) & $46 \%$ & $61 \%$ & 0.4132 \\
Mean PASI & NA & $11.1 \pm 8.9$ & NA \\
Median PASI & NA & 7.75 & NA
\end{tabular}

PASI Psoriasis Area and Severity Index 
Table 2 Sample composition

\begin{tabular}{llllllll}
\hline Skin site & Arm & Axilla & Scalp & Trunk & Gluteal fold & Leg & Sum \\
\hline Skin type & Dry & Moist & Sebaceous & Dry & Moist & Dry & - \\
Healthy & 26 & 19 & 25 & 26 & 25 & 26 & 147 \\
PSO_L & 22 & 8 & 23 & 17 & 15 & 27 & 112 \\
PSO_N & 27 & 24 & 25 & 27 & 27 & 28 & 158 \\
\hline
\end{tabular}

psoriasis patients (PSO_L). We sampled all six sites for both healthy (Healthy) and unaffected skin (PSO_N). Only sites with psoriasis lesions present were sampled for psoriatic lesional samples (PSO_L). The psoriasis subjects in our cohort most frequently had psoriatic plaques on the arms, legs, and scalp, whereas there was lowest frequency in the axilla (armpit). Intermediate frequency of plaques was found on the trunk and gluteal fold (Table 2). The sampling of these six skin sites from psoriatic lesional skin, psoriatic non-lesional skin, and healthy control skin allowed for an examination of how the psoriatic microbiome differs at different sites as well as how it changes with disease progression (lesional vs non-lesional).

\section{Alteration in psoriatic skin microbiome diversity is site specific and exhibits an increasing trend in alpha diversity and greater heterogeneity compared with healthy skin}

The diversity of the microbial community in a given human body site reflects the structure and composition of the community. Alterations in human microbiome diversity has been linked to disease states. For example, reduced bacterial alpha diversity in the gut microbiome has been linked to obesity and inflammatory bowel disease (IBD) [27-29] while increased diversity in the vaginal microbiome is associated with bacterial vaginosis $[30,31]$. To understand if diversity of the skin microbial community is altered in psoriasis patients, we first examined alpha diversity of psoriatic lesional skin, psoriatic unaffected skin and healthy control skin using four different metrics to measure community richness (chao1 and observed OTUs), evenness (Simpson diversity index) and overall diversity (Shannon index) (Fig. 1a-d, and Table 3a). Overall, we observed increasing diversity in all four measures going from healthy skin to non-lesional skin to lesional skin, with a statistically significant trend for the Simpson (Fig. 1c, $p$-value $=0.005$ ) and Shannon indices (Fig. $1 \mathrm{~d}, p$-value $=0.005$ ). This unidirectional trend in microbiome diversity suggests that the skin microbiome community diversifies as psoriatic disease progresses. To evaluate alpha diversity at different skin sites, we further examined the four metrics at each skin site. Interestingly, we found significantly increased community richness (chao1) in scalp psoriatic lesions compared to healthy scalp and increased community evenness (Simpson and Shannon indices) in arm psoriatic lesional and non-lesional skin compared to arm healthy control skin, with a significant trend test in the arm for the Simpson and Shannon indices (Table 4). When we grouped samples by skin type (Table 5), we observed higher alpha diversity in all four indices at dry psoriatic skin sites (arm, leg, trunk combined) relative to healthy skin, but no difference in alpha diversity for moist sites (axilla, gluteal fold combined). Overall, these results indicate that increased alpha diversity in psoriasis is mostly observed at dry skin sites, with a trend at the sebaceous (scalp) site, and no increase at moist sites. Our data demonstrates that the association between skin microbiome and psoriasis is complex and sometimes site and/or skin type specific. This highlights the need for comprehensive sampling at various skin sites and skin types to study the skin microbiome in association with cutaneous disease.

We further explored the relationship among bacterial communities isolated from psoriatic and healthy skin by calculating beta diversity using weighted Unifrac distance [32]. There was no distinct difference between bacterial communities isolated from the healthy skin and psoriatic skin as there was not a distinctive separation between bacterial communities isolated according to skin status (Fig. 1c) and the first PC is not significantly different in both psoriasis disease states and healthy skin (PSO_L vs Healthy: $p$ value $=0.109$, PSO_N vs. Healthy: $p$ value $=0.128$ ). Although we did not observe distinct clusters associated with disease states, the bacterial communities isolated from psoriatic skin were more dispersed in the principal coordinate analysis than those from healthy skin (Fig. 1e). Indeed, we assess the community dispersion of each disease status by calculating the mean weighted Unifrac distance between bacterial microbiota found in either healthy, psoriatic non-lesional or psoriatic lesional skin and noted that psoriatic non-lesional skin or psoriatic lesional skin exhibited significantly higher mean distances compared with healthy skin (Fig. 1f), indicating greater heterogeneity in the composition of skin microbiota of psoriatic patients irrespective of lesions. We observed a similar trend of increasing heterogeneity by disease state in skin bacterial communities isolated from the arm, trunk, and leg (Additional file 1: Figure S1A, S1B, S1C) as well as in the dry skin group (Additional file 1: Figure S2A). In the moist skin group, bacterial communities of psoriasis lesional skin also exhibited higher heterogeneity compared to healthy and non-lesional skin (Additional file 1: Figure S2B). The heterogeneity differences in moist skin group were largely driven by samples from the gluteal fold (Additional file 1: Figure S1E), as there was little difference in heterogeneity for the axilla (Additional file 1: Figure S1D). Interestingly, the scalp skin microbiome displayed no differences in heterogeneity among different disease states. These results indicate increasing beta heterogeneity for all dry skin sites in psoriasis and for the gluteal fold in psoriasis. Taken with the previous results for alpha diversity, there appears to be an overall loss of stability in the skin microbial community as psoriatic disease progresses, particularly for dry skin sites. 
A

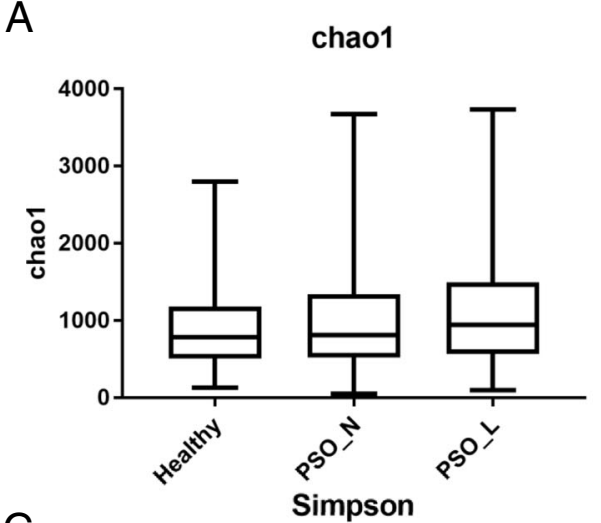

C
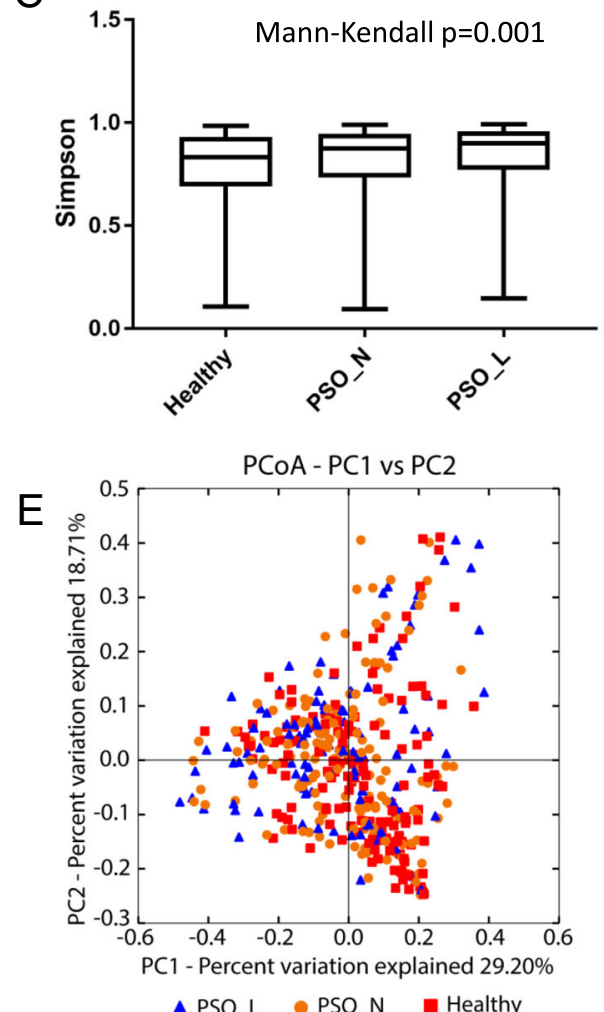

B

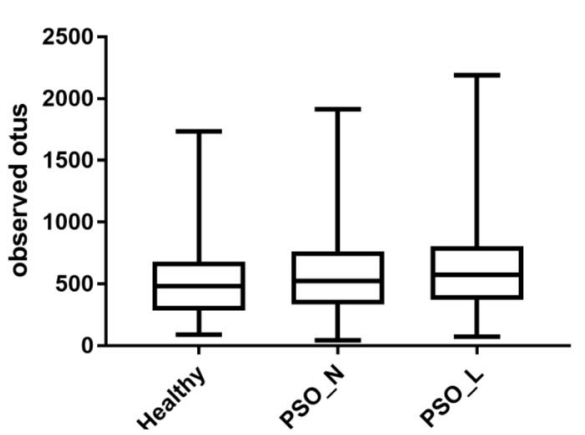

$\mathrm{D}$

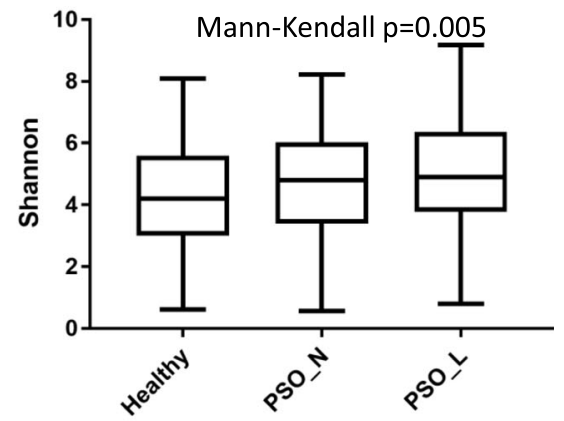

F

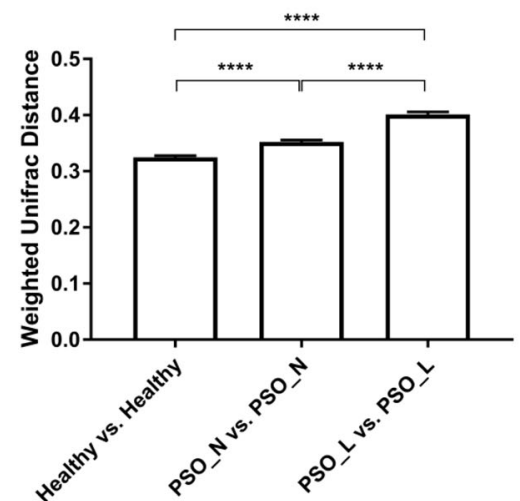

Fig. 1 Bacterial community diversity in healthy and psoriasis skin. Alpha diversity measured according to a chao1 index, b observed OTUs, c Simpson's diversity index, and $\mathbf{d}$ Shannon index of healthy skin samples, psoriasis non-lesional samples, and psoriasis lesional skin samples. Significant trends of alpha diversity are identified by a Mann-Kendall trend test with $p$-value shown. e Principal coordinate analysis (PCoA) of the microbial community structures based on weighted UniFrac distance matrix for the first two principal axes. Each point on the PCoA plot represents a skin microbiome sample (red square $=$ healthy, blue triangle $=$ psoriasis lesional, and orange circle = psoriasis unaffected). The first principal coordinate explains $29.6 \%$ of variation, and the second principal coordinate explains $18.70 \%$ of the variation. $\mathbf{f}$ The average weighted UniFrac distances among samples within each disease state are shown in the box plot. The samples in the psoriatic lesional group are more heterogeneous than samples from healthy or psoriasis unaffected groups (one-way ANOVA with Tukey correction, ${ }^{* * *} p$ value $<0.0001$ )

Table 3 Summary of alpha diversity according to disease status

\begin{tabular}{llllllll}
\hline Alpha diversity metrics & Healthy.mean & Healthy.std & PSON.mean & PSON.std & PSOL.mean & PSOL.std & $p$ value (trend) \\
\hline Chao1 & 891.86 & 534.25 & 1012.74 & 654.40 & 1090.78 & 650.49 & 0.18 \\
Observed OTU & 521.77 & 314.82 & 572.43 & 324.16 & 614.92 & 346.02 & 0.15 \\
Shannon & 4.33 & 1.72 & 4.68 & 1.70 & 4.94 & 1.71 & 0.005 \\
Simpson & 0.77 & 0.20 & 0.81 & 0.19 & 0.84 & 0.17 & 0.00097 \\
\hline
\end{tabular}


Table 4 Summary of alpha diversity within each skin site

\begin{tabular}{|c|c|c|c|c|c|c|c|c|}
\hline Site & Alpha diversity metrics & Healthy.mean & Healthy.std & PSON.mean & PSON.std & PSOL.mean & PSOL.std & $p$ value (trend) \\
\hline Scalp & Chaol & 626.17 & 378.94 & 794.56 & 561.43 & $939.86^{*}$ & 617.36 & 0.06 \\
\hline Scalp & Observed OTU & 335.72 & 217.12 & 407.84 & 287.03 & 478.3 & 297.17 & 0.07 \\
\hline Scalp & Shannon & 3.18 & 1.5 & 3.38 & 1.68 & 3.77 & 1.59 & 0.26 \\
\hline Scalp & Simpson & 0.68 & 0.21 & 0.68 & 0.21 & 0.74 & 0.17 & 0.47 \\
\hline Arm & Chao1 & 1024.07 & 438.77 & 1381.73 & 742.52 & 1255.45 & 680.35 & 0.19 \\
\hline Arm & Observed OTU & 594.58 & 220.89 & 772.44 & 328.77 & 755.73 & 400.6 & 0.11 \\
\hline Arm & Shannon & 4.43 & 1.42 & $5.44^{*}$ & 1.44 & $5.66^{* *}$ & 1.54 & 0.007 \\
\hline Arm & Simpson & 0.76 & 0.17 & $0.86^{*}$ & 0.14 & $0.89^{*}$ & 0.11 & 0.002 \\
\hline Leg & Chao1 & 1169.15 & 558.4 & 1351.63 & 780.77 & 1356.34 & 735.23 & 0.4 \\
\hline Leg & Observed OTU & 687.62 & 300.84 & 764.07 & 359.84 & 763.3 & 364.11 & 0.37 \\
\hline Leg & Shannon & 5.37 & 1.29 & 5.68 & 1.43 & 5.49 & 1.7 & 0.59 \\
\hline Leg & Simpson & 0.89 & 0.08 & 0.89 & 0.11 & 0.86 & 0.17 & 0.54 \\
\hline Trunk & Chao1 & 880.93 & 506.25 & 950.95 & 537.29 & 1038.19 & 469.78 & 0.28 \\
\hline Trunk & Observed OTU & 518.85 & 312.88 & 540.48 & 277.26 & 609.47 & 238.09 & 0.17 \\
\hline Trunk & Shannon & 4.07 & 2.07 & 4.32 & 1.87 & 5.19 & 1.42 & 0.09 \\
\hline Trunk & Simpson & 0.69 & 0.27 & 0.75 & 0.25 & 0.86 & 0.14 & 0.07 \\
\hline Axilla & Chao1 & 610.81 & 489.85 & 676.19 & 459.89 & 544.68 & 475.22 & 0.89 \\
\hline Axilla & Observed OTU & 357.21 & 283.61 & 390.33 & 225.85 & 285.88 & 175.58 & 0.82 \\
\hline Axilla & Shannon & 3.78 & 1.49 & 3.99 & 1.19 & 3.62 & 1.15 & 0.19 \\
\hline Axilla & Simpson & 0.76 & 0.16 & 0.78 & 0.16 & 0.78 & 0.24 & 0.22 \\
\hline Gluteal fold & Chao1 & 956.62 & 572.91 & 855.25 & 354.03 & 953.51 & 412.17 & 0.78 \\
\hline Gluteal fold & Observed OTU & 587.72 & 366.3 & 519.89 & 193.96 & 532.47 & 204.86 & 0.85 \\
\hline Gluteal fold & Shannon & 5.01 & 1.38 & 5.04 & 1.23 & 5.12 & 1.36 & 0.91 \\
\hline Gluteal fold & Simpson & 0.85 & 0.12 & 0.87 & 0.1 & 0.88 & 0.1 & 0.22 \\
\hline
\end{tabular}

${ }^{*} p$ value $<0.05$ compared to healthy, ${ }^{* *} p$ value $<0.01$ compared to healthy

Psoriasis skin microbiota is enriched for Staphylococcus aureus and Staphylococcus pettenkoferi

We next examined bacterial composition of skin microbial communities from psoriatic and healthy skin at various taxonomic levels. The skin microbiome of all disease states (healthy, psoriatic lesional, and psoriatic non-lesional) consisted of four dominant phyla: Actinobacteria (53.8-66.5\%) Firmicutes (23.9-28.3\%), Proteobacteria (5.8-12.0\%), and Bacteroidetes (2.1-2.9\%) (Fig. 2a), consistent with previous descriptions of skin microbiota composition [4]. At the genus level, skin microbiome is dominated by Propionibacterium (22.8-38.1\%), Corynebacterium (21.4-23.9\%), Staphylococcus (5.3-9.2\%) in all disease states (Fig. 2b). Although the dominant taxa are similar in different disease states, we observed a gradual shift of taxonomic composition from healthy skin to psoriatic non-lesional skin to psoriatic lesional skin at both phylum and genus levels, suggesting that these microbial

Table 5 Summary of alpha diversity according to skin type

\begin{tabular}{lllllllll}
\hline Skin type & Metrics & Healthy.mean & Healthy.std & PSON.mean & PSON.std & PSOL.mean & PSOL.std & $p$ value (trend) \\
\hline Dry & Chao1 & 1024.72 & 517.09 & 1229.61 & 723.2 & 1240.76 & 669.61 & 0.05 \\
Dry & Observed OTU & 600.35 & 289.53 & 693.21 & 341.44 & 721.15 & 355.91 & 0.02 \\
Dry & Shannon & 4.62 & 1.72 & 5.15 & 1.7 & 5.47 & 1.59 & 0.004 \\
Dry & Simpson & 0.78 & 0.21 & 0.83 & 0.19 & 0.87 & 0.15 & 0.001 \\
Moist & Chao1 & 807.29 & 565.2 & 770.99 & 416.98 & 811.31 & 476.72 & 0.89 \\
Moist & Observed OTU & 488.18 & 352.14 & 458.92 & 219.32 & 446.7 & 227.79 & 0.86 \\
Moist & Shannon & 4.48 & 1.55 & 4.55 & 1.32 & 4.6 & 1.48 & 0.5 \\
Moist & Simpson & 0.81 & 0.15 & 0.83 & 0.14 & 0.85 & 0.17 & 0.25 \\
\hline
\end{tabular}

Dry = arm, leg, trunk; moist = axilla, gluteal fold 


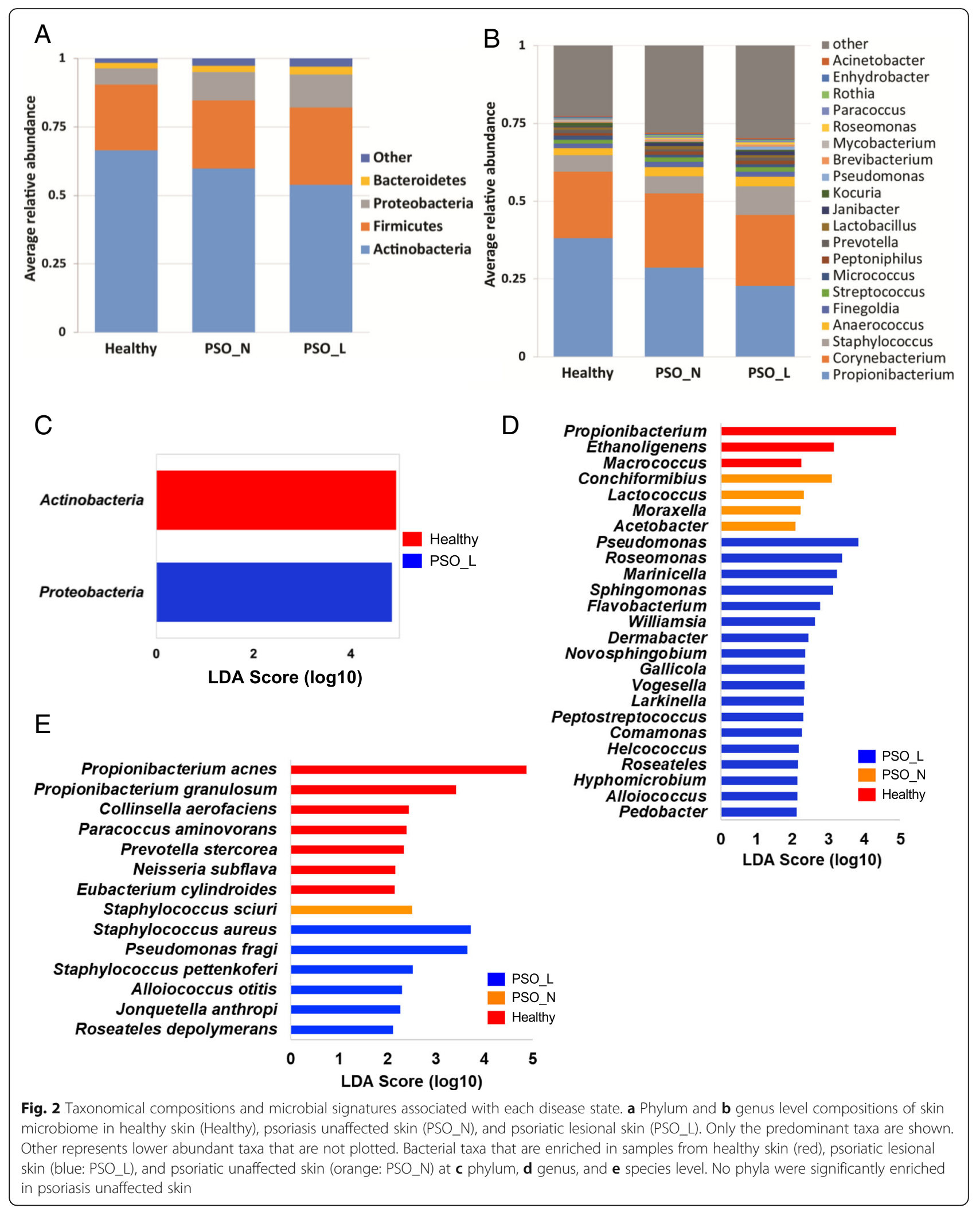


Table 6 Microbial genera associated with different skin status

\begin{tabular}{|c|c|c|c|c|}
\hline Feature & Log(highest_class_avg) & Class enriched & LDA effect size & $p$ value \\
\hline g_Propionibacterium & 5.58 & Healthy & 4.88 & $1.54 \mathrm{E}-04$ \\
\hline g_Ethanoligenens & 0.61 & Healthy & 3.16 & $2.47 \mathrm{E}-02$ \\
\hline g__Macrococcus & 2.60 & Healthy & 2.25 & $2.37 \mathrm{E}-02$ \\
\hline g_Pseudomonas & 3.99 & PSO_L & 3.83 & $2.04 \mathrm{E}-04$ \\
\hline g_Roseomonas & 3.77 & PSO_L & 3.38 & $3.35 E-02$ \\
\hline g_Marinicella & 1.11 & PSO_L & 3.24 & $1.65 \mathrm{E}-02$ \\
\hline g__sphingomonas & 3.54 & PSO_L & 3.14 & $5.05 \mathrm{E}-03$ \\
\hline g_Flavobacterium & 3.16 & PSO_L & 2.78 & $2.87 \mathrm{E}-05$ \\
\hline f__Flavobacteriaceae_Other & 1.03 & PSO_L & 2.67 & 4.15E-03 \\
\hline g__Williamsia & 3.05 & PSO_L & 2.63 & 4.97E-02 \\
\hline f__Micrococcaceae_Other & 3.23 & PSO_L & 2.60 & 8.07E-03 \\
\hline g__Dermabacter & 3.25 & PSO_L & 2.45 & $3.27 \mathrm{E}-03$ \\
\hline g__Novosphingobium & 3.00 & PSO_L & 2.36 & $8.24 \mathrm{E}-03$ \\
\hline g_gallicola & 2.68 & PSO_L & 2.34 & $3.96 \mathrm{E}-02$ \\
\hline g_Vogesella & 2.50 & PSO_L & 2.34 & $2.36 \mathrm{E}-02$ \\
\hline g_Larkinella & 1.16 & PSO_L & 2.32 & $6.12 \mathrm{E}-03$ \\
\hline g__Peptostreptococcus & 2.86 & PSO_L & 2.29 & $4.72 \mathrm{E}-02$ \\
\hline g__Comamonas & 2.67 & PSO_L & 2.27 & $2.11 \mathrm{E}-02$ \\
\hline f_Rhodocyclaceae_Other & 2.78 & PSO_L & 2.22 & 4.96E-02 \\
\hline g__Helcococcus & 2.61 & PSO_L & 2.17 & $9.83 \mathrm{E}-04$ \\
\hline g__Roseateles & 0.83 & PSO_L & 2.16 & $2.08 \mathrm{E}-02$ \\
\hline g_Hyphomicrobium & 2.56 & PSO_L & 2.13 & 1.86E-02 \\
\hline g_Alloiococcus & 2.54 & PSO_L & 2.13 & 1.43E-06 \\
\hline g_Pedobacter & 2.60 & PSO_L & 2.13 & 1.90E-02 \\
\hline g_Conchiformibius & 3.28 & PSO_N & 3.10 & $6.96 \mathrm{E}-04$ \\
\hline f_Bradyrhizobiaceae_Other & 2.99 & PSO_N & 2.66 & $2.30 \mathrm{E}-02$ \\
\hline g_Lactococcus & 2.98 & PSO_N & 2.32 & $1.58 \mathrm{E}-02$ \\
\hline g__Moraxella & 2.55 & PSO_N & 2.22 & $4.54 \mathrm{E}-03$ \\
\hline g_Acetobacter & 2.25 & PSO_N & 2.08 & $2.59 \mathrm{E}-03$ \\
\hline
\end{tabular}

community shifts may precede the appearance of lesions in patients and have potential roles in disease progression. To further associate the taxonomic shift to different disease states, we identified bacterial taxa that discriminate each disease group using Lefse [33]. At the phylum level, Actinobacteria and Proteobacteria served as strong discriminants for the skin microbiome from healthy and psoriatic lesions respectively (Fig. 2c). Lefse identified three genera, Propionibacterium, Ethanoligenens, and Macrococcus, as additional discriminative signatures for healthy skin (Fig. 2d). Lefse also identified 18 microbial genera that are discriminatively associated with psoriatic lesional skin including the genus Pseudomonas, which includes many opportunistic pathogens (Fig. 2d, Table 6). Four genera, Conchiformibius, Lactococcus, Moraxella, and Acetobacter, were associated with psoriatic unaffected skin (Fig. 2d). The combination of these genera can serve as potential markers for distinguishing skin from different disease states.

Our 16S rRNA sequencing also provided species-level resolution for some but not all of the sequencing reads. Lefse analysis identified several species-level bacterial signatures specific for different disease states (Fig. 2e). Consistent with what we observed in the genus level, the healthy skin microbiome was more enriched in both Propionibacterium acnes (P. acnes) and Propionibacterium granulosum ( $P$. granulosum) compared to the psoriasis-associated skin microbiome (Fig. 3a, b). Staphylococcus sciuri was enriched in psoriatic non-lesional skin (Fig. 3c). Interestingly, two Staphylococcus species, S. aureus and S. pettenkoferi were significantly enriched in the psoriatic lesions while the genera Staphylococcus as a whole was not significantly enriched with any skin condition in our analysis (Fig. 2d, e). 

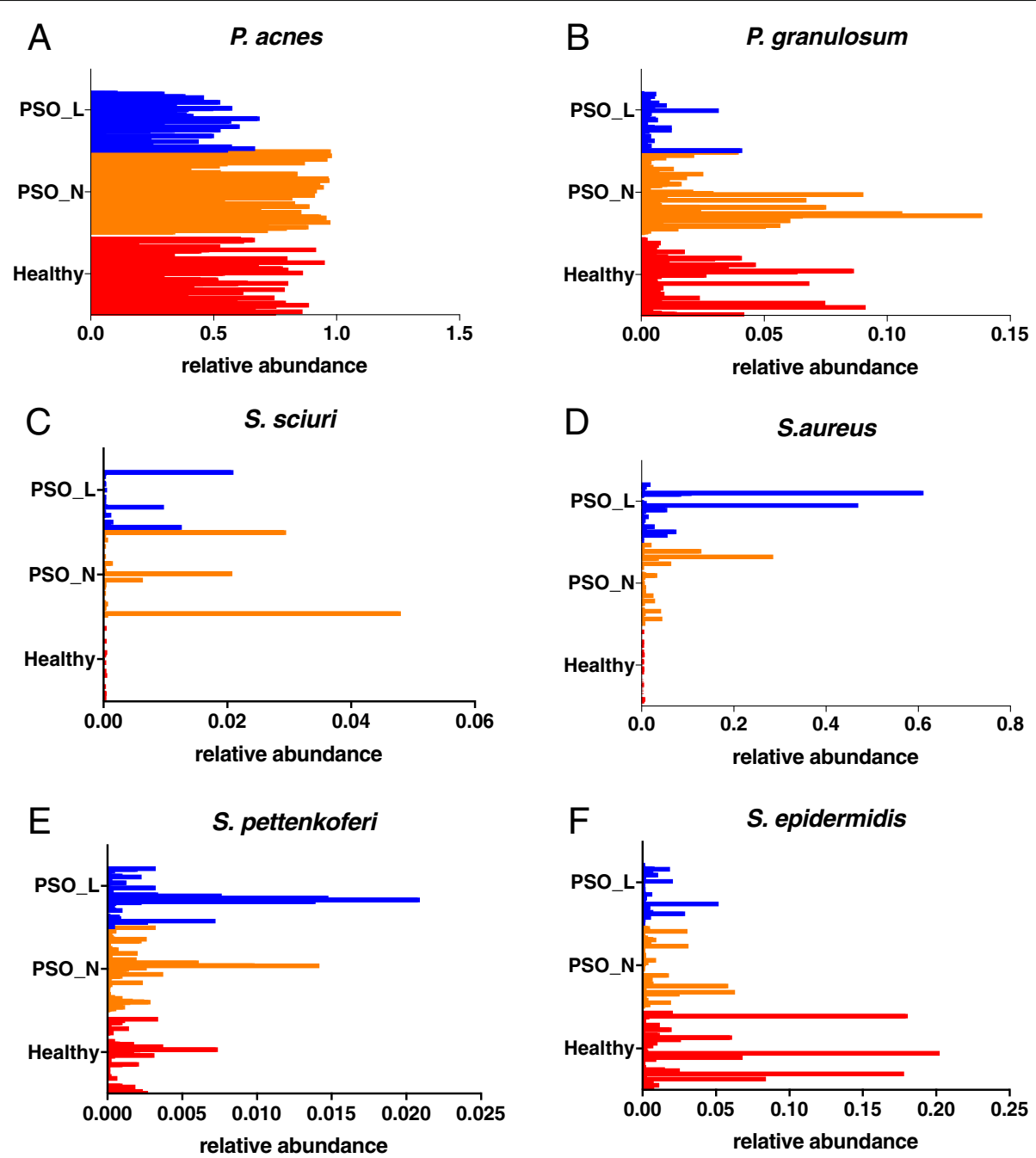

Fig. 3 Relative abundance of bacterial species in each disease state. Histograms represent the relative abundances of specific bacterial species in samples from healthy skin (red bars: Healthy), psoriatic lesions (blue bars: PSO_L), and psoriatic unaffected skin (orange bars: PSO_N). Samples from healthy skin and psoriatic unaffected skin are more abundant in a Propionibacterium acnes ( $p$ value $=0.0002 ;$ LDA effect size $=4.87$ ) and b Propionibacterium granulosum ( $p$ value $=0.014 ;$ LDA effect size $=3.41$ ). Samples from psoriatic skin (both unaffected and lesional) are more abundant in c Staphylococcus sciuri ( $p$ value $=0.032$; LDA effect size $=2.51$ ), $\mathbf{d}$ Staphylococcus aureus ( $p$ value $=0.007$; LDA effect size $=3.72$ ), and e Staphylococcus pettenkoferi ( $p$ value $=0.012$; LDA effect size $=2.52$ ). On the contrary, $\mathbf{f}$ Staphylococcus epidermidis shows a trend of increased abundance in healthy skin but the difference did not reach the statistical significance

We further explored the relative abundance of the Staphylococcus species across all samples with different disease states (Table 7). Strikingly, Staphylococcus aureus was more abundant in both lesional and non-lesional psoriatic skin compared to healthy skin (Fig. 3d). Although a low level of $S$. aureus was detected in 102 out of 147 healthy control samples and was detected in at least one skin swab of every healthy control subject, increased S. aureus abundance was exclusively observed in psoriasis samples (Fig. 3d). A similar trend was observed for Staphylococcus pettenkoferi, although to a lesser degree (Fig. 3e). In contrast, Staphylococcus epidermidis was more abundant in healthy skin compared to psoriatic skin (Fig. 3f) which is consistent with the previously reported competitive relationship between the Staphylococcus epidermidis and Staphylococcus aureus [34]. The dynamic inter-microbe relationship between different Staphylococcus species might contribute to the distinct microbial communities associated with healthy and psoriatic skin.

Anatomic skin site is one of the major determinants of skin microbiome composition [24, 35]. Therefore, we further used Lefse to identify bacterial species at each skin site associated with healthy, non-lesional psoriatic, and lesional psoriatic skin (Table 8). We found that a reduced abundance of $P$. acnes is associated with psoriasis lesional skin at the arm, trunk, and gluteal fold (Additional file 1: Figure S3A), with a similar trend for 
Table 7 Microbial species associated with different skin status

\begin{tabular}{|c|c|c|c|c|}
\hline Feature & Log(highestClassAvg) & Class & LDA effect size & $p$ value \\
\hline f_Propionibacteriaceae_g_Propionibacterium_s_acnes & 5.57 & Healthy & 4.87 & $\overline{1.92 \mathrm{E}-04}$ \\
\hline f__Propionibacteriaceae_g__Propionibacterium_s_granulosum & 3.96 & Healthy & 3.41 & 1.36E-02 \\
\hline f_Coriobacteriaceae_g_Atopobium_other & 3.32 & Healthy & 2.93 & $6.00 \mathrm{E}-03$ \\
\hline f__Ruminococcaceae_g_Ethanoligenens_s__ & 0.61 & Healthy & 2.66 & 2.47E-02 \\
\hline f__Gracilibacteraceae_g__s__ & 0.67 & Healthy & 2.48 & 1.76E-02 \\
\hline f_Coriobacteriaceae_g_Collinsella_s_aerofaciens & 2.89 & Healthy & 2.44 & 1.09E-02 \\
\hline f_Sphingomonadaceae_g__Novosphingobium_s__ & 2.99 & Healthy & 2.43 & 1.07E-02 \\
\hline f__Rhodobacteraceae_g_Paracoccus_s_aminovorans & 2.80 & Healthy & 2.39 & 1.00E-02 \\
\hline f_Prevotellaceae_g__Prevotella_s__stercorea & 2.60 & Healthy & 2.33 & $1.71 \mathrm{E}-03$ \\
\hline f_Desulfovibrionaceae_g__Desulfovibrio_s__ & 1.16 & Healthy & 2.24 & 2.47E-02 \\
\hline f__Rickettsiaceae_g_Rickettsia_s__ & 1.40 & Healthy & 2.22 & 2.83E-03 \\
\hline f_Victivallaceae_g__s_ & 1.04 & Healthy & 2.20 & 6.97E-03 \\
\hline f__Rivulariaceae_other_other & 1.06 & Healthy & 2.19 & 2.47E-02 \\
\hline f_Erysipelotrichaceae_g_cc_115_s_ & 0.94 & Healthy & 2.16 & $1.71 \mathrm{E}-02$ \\
\hline f__Neisseriaceae_g_Neisseria_s_subflava & 2.68 & Healthy & 2.16 & 1.83E-02 \\
\hline f_Erysipelotrichaceae_g__Eubacterium_s_cylindroides & 2.34 & Healthy & 2.15 & $1.71 \mathrm{E}-02$ \\
\hline f__Succinivibrionaceae_g_Succinivibrio_s__ & 1.88 & Healthy & 2.06 & 5.69E-04 \\
\hline f_Lachnospiraceae_g__Coprococcus_other & 1.55 & Healthy & 2.01 & 6.91E-08 \\
\hline f_Staphylococcaceae_g_Staphylococcus_s__aureus & 4.16 & PSO_L & 3.72 & 7.47E-03 \\
\hline f_Pseudomonadaceae_g_Pseudomonas_s_fragi & 3.93 & PSO_L & 3.64 & 3.35E-04 \\
\hline f__Methylobacteriaceae_g__s_ & 3.81 & PSO_L & 3.38 & $1.68 \mathrm{E}-03$ \\
\hline f__oxalobacteraceae_g__s__ & 3.69 & PSO_L & 3.27 & 4.40E-03 \\
\hline f_flavobacteriaceae_other_other & 1.03 & PSO_L & 2.94 & 4.15E-03 \\
\hline f__Sphingomonadaceae_g_Sphingomonas_s__ & 3.41 & PSO_L & 2.94 & $2.08 \mathrm{E}-02$ \\
\hline o_thiohalorhabdales_f__g_s_ & 1.40 & PSO_L & 2.89 & 4.15E-03 \\
\hline f_Ellin517_g__s_ & 0.48 & PSO_L & 2.85 & $2.28 \mathrm{E}-02$ \\
\hline f__Marinicellaceae_g_Marinicella_s__ & 1.11 & PSO_L & 2.85 & 1.65E-02 \\
\hline f_Propionibacteriaceae_g__Tessaracoccus_s__ & 0.78 & PSO_L & 2.79 & 2.76E-02 \\
\hline$f \_g \_s-$ & 1.08 & PSO_L & 2.77 & $2.28 \mathrm{E}-02$ \\
\hline f_flavobacteriaceae_g_flavobacterium_s__ & 3.12 & PSO_L & 2.69 & $5.19 \mathrm{E}-05$ \\
\hline f__Micrococcaceae_other_other & 3.23 & PSO_L & 2.69 & 9.67E-03 \\
\hline f_Williamsiaceae_g__Williamsia_s__ & 3.05 & PSO_L & 2.68 & 4.36E-02 \\
\hline f__Microbacteriaceae_g__s_ & 3.27 & PSO_L & 2.67 & $2.24 \mathrm{E}-02$ \\
\hline f_Xanthomonadaceae_g__Wohlfahrtiimonas_s__ & 1.16 & PSO_L & 2.66 & 1.65E-02 \\
\hline f_Cytophagaceae_g_Larkinella_s__ & 1.16 & PSO_L & 2.60 & $6.02 \mathrm{E}-03$ \\
\hline f__Pseudomonadaceae_g_Pseudomonas_s__ & 3.06 & PSO_L & 2.57 & 2.33E-03 \\
\hline f_Rhodobacteraceae_g_Anaerospora_other & 1.16 & PSO_L & 2.52 & $5.25 E-03$ \\
\hline f__Staphylococcaceae_g_Staphylococcus_s__pettenkoferi & 2.99 & PSO_L & 2.52 & 1.23E-02 \\
\hline f_Comamonadaceae_g_Limnobacter_s__ & 1.55 & PSO_L & 2.51 & 4.31E-02 \\
\hline f__Kineosporiaceae_g_Kineosporia_s__ & 1.26 & PSO_L & 2.49 & 3.75E-02 \\
\hline f_Legionellaceae_g_Legionella_other & 1.60 & PSO_L & 2.46 & 1.15E-02 \\
\hline f_Chitinophagaceae_g__s_ & 2.81 & PSO_L & 2.44 & 4.84E-04 \\
\hline f_Acetobacteraceae_g__s_ & 3.11 & PSO_L & 2.42 & $1.75 E-02$ \\
\hline f_frankiaceae_other_other & 1.46 & PSO_L & 2.42 & $1.91 \mathrm{E}-03$ \\
\hline
\end{tabular}


Table 7 Microbial species associated with different skin status (Continued)

\begin{tabular}{|c|c|c|c|c|}
\hline Feature & Log(highestClassAvg) & Class & LDA effect size & $p$ value \\
\hline f_Ectothiorhodospiraceae_g__s__ & 1.67 & PSO_L & 2.36 & 1.85E-02 \\
\hline f__Dermabacteraceae_g_Dermabacter_s__ & 3.25 & PSO_L & 2.34 & 2.79E-03 \\
\hline f__Coxiellaceae_g__s_ & 1.60 & PSO_L & 2.31 & 2.62E-02 \\
\hline f__Tissierellaceae_g_Gallicola_s__ & 2.68 & PSO_L & 2.30 & 3.94E-02 \\
\hline f__Aerococcaceae_g_Alloiococcus_s_otitis & 2.41 & PSO_L & 2.30 & 1.04E-05 \\
\hline f__Hyphomicrobiaceae_g__Hyphomicrobium_s__ & 2.52 & PSO_L & 2.28 & 2.71E-02 \\
\hline f__Dethiosulfovibrionaceae_g__Jonquetella_s_anthropi & 0.99 & PSO_L & 2.26 & $2.08 \mathrm{E}-02$ \\
\hline f__Rhodospirillaceae_g__s_ & 2.66 & PSO_L & 2.26 & 1.26E-02 \\
\hline f_CComamonadaceae_g_CComamonas_s__ & 2.54 & PSO_L & 2.24 & 4.73E-03 \\
\hline f__Piscirickettsiaceae_g__s_ & 1.78 & PSO_L & 2.23 & 4.39E-03 \\
\hline f_Methylobacteriaceae_g__Methylobacterium_s__ & 2.86 & PSO_L & 2.21 & 1.00E-02 \\
\hline f__Tissierellaceae_g__Helcococcus_s__ & 2.61 & PSO_L & 2.21 & 1.05E-03 \\
\hline f_Comamonadaceae_g_Rhodoferax_s__ & 1.42 & PSO_L & 2.18 & 1.39E-02 \\
\hline f__Neisseriaceae_g_Vogesella_s__ & 2.50 & PSO_L & 2.18 & $2.42 \mathrm{E}-02$ \\
\hline f_Leuconostocaceae_g__Weissella_other & 1.33 & PSO_L & 2.17 & 9.64E-03 \\
\hline f_Alcaligenaceae_g_oligella_s__ & 2.34 & PSO_L & 2.12 & 3.31E-02 \\
\hline f_Erythrobacteraceae_g__s_ & 2.67 & PSO_L & 2.12 & $2.31 \mathrm{E}-03$ \\
\hline f__Beijerinckiaceae_g__s_ & 2.54 & PSO_L & 2.12 & 1.62E-02 \\
\hline f__Comamonadaceae_g__Roseateles_s_depolymerans & 0.83 & PSO_L & 2.12 & $2.08 \mathrm{E}-02$ \\
\hline f_Bacillaceae_other_other & 2.27 & PSO_L & 2.07 & 8.81E-04 \\
\hline o__Phycisphaerales_f__ g__s_ & 1.59 & PSO_L & 2.04 & 3.64E-02 \\
\hline f__Neisseriaceae_g__s_ & 4.28 & PSO_N & 3.78 & 2.89E-02 \\
\hline f_Neisseriaceae_g_Conchiformibius_s__ & 3.28 & PSO_N & 3.08 & 4.83E-05 \\
\hline f_Moraxellaceae_g_Acinetobacter_other & 3.26 & PSO_N & 2.95 & $9.18 \mathrm{E}-03$ \\
\hline f__Micrococcaceae_g__s_ & 3.34 & PSO_N & 2.81 & 5.11E-03 \\
\hline f_Bradyrhizobiaceae_other_other & 2.99 & PSO_N & 2.70 & 2.23E-02 \\
\hline f_Staphylococcaceae_g_Staphylococcus_s_sciuri & 2.85 & PSO_N & 2.51 & $3.23 \mathrm{E}-02$ \\
\hline f__Syntrophobacteraceae_g__s__ & 1.26 & PSO_N & 2.40 & 2.85E-02 \\
\hline f_Streptococcaceae_g_LLactococcus_s__ & 2.98 & PSO_N & 2.38 & 3.17E-02 \\
\hline f___Chthoniobacteraceae_g__s_ & 1.26 & PSO_N & 2.35 & $2.55 \mathrm{E}-02$ \\
\hline f_Moraxellaceae_g__Moraxella_s__ & 2.55 & PSO_N & 2.29 & $1.29 \mathrm{E}-03$ \\
\hline f_Moraxellaceae_g_Perlucidibaca_s__ & 2.06 & PSO_N & 2.25 & 2.62E-02 \\
\hline f_Actinomycetaceae_g__Trueperella_s__ & 1.01 & PSO_N & 2.22 & 3.63E-02 \\
\hline f__Pseudonocardiaceae_g___s_ & 1.09 & PSO_N & 2.08 & 2.21E-02 \\
\hline
\end{tabular}

the scalp and axilla. We did not observe a decrease for $P$. acnes in leg psoriasis samples, which is possibly due to the low abundance of $P$. acnes in healthy leg skin (Additional file 1: Figure S3A). Together, our data suggest that $P$. acnes may play a crucial role to maintain skin health at most skin sites besides the leg. Surprisingly, we did not observe a statistically significant increase in $S$. aureus abundance in psoriasis compared to healthy skin at any individual skin site (Table 8), whereas when anatomic sites were combined, $S$. aureus was highly associated with psoriasis lesional skin (Figs. 2e and $3 \mathrm{~d}$ ). Therefore, we defined a group of psoriasis samples with $S$. aureus abundance above the highest level of $S$. aureus colonization in healthy skin (baseline level $=0.0068$ ) as "S. aureus high samples" (Table 9). We found that $S$. aureus high samples were observed exclusively in psoriasis patients and were seen at all skin sites (Additional file 1: Figure S3C), but that the number of $S$. aureus high samples at each skin site is modest, between 2 and 8 (Additional file 1: Figure S3D). This 
Table 8 Microbial species associated with different disease state in each skin site

\begin{tabular}{|c|c|c|c|c|}
\hline Feature & Log(highest_class_avg) & Class enriched & LDA effect size & $p$ value \\
\hline \multicolumn{5}{|l|}{ Arm } \\
\hline Propionibacterium_acnes & 5.67 & Healthy & 5.02 & 0.001 \\
\hline Leadbetterella_s__ & 1.54 & PSO_L & 2.25 & 0.024 \\
\hline Comamonas_s__ & 2.94 & PSO_L & 2.59 & 0.041 \\
\hline Acinetobacter_Other & 3.44 & PSO_L & 3.13 & 0.048 \\
\hline Vogesella_s__ & 3.20 & PSO_L & 3.03 & 0.047 \\
\hline Conchiformibius_s__ & 4.02 & PSO_N & 3.61 & 0.047 \\
\hline Pseudomonas_s__ & 3.30 & PSO_N & 2.83 & 0.035 \\
\hline Peptococcus_s__ & 2.26 & PSO_N & 2.00 & 0.019 \\
\hline Euzebya_s__ & 2.14 & PSO_N & 2.03 & 0.038 \\
\hline \multicolumn{5}{|l|}{ Trunk } \\
\hline Propionibacterium_s_acnes & 5.68 & Healthy & 5.19 & 0.004 \\
\hline Moraxella_s__ & 2.51 & PSO_L & 2.29 & 0.006 \\
\hline Helcococcus_s__ & 2.50 & PSO_L & 2.14 & 0.006 \\
\hline Conchiformibius_s__ & 2.53 & PSO_N & 2.26 & 0.027 \\
\hline \multicolumn{5}{|l|}{ Leg } \\
\hline Xanthobacter_s__ & 2.31 & Healthy & 2.06 & 0.011 \\
\hline Flavobacterium_s__ & 2.93 & PSO_L & 2.38 & 0.028 \\
\hline Pseudomonas_s_fragi & 3.25 & PSO_L & 2.88 & 0.036 \\
\hline Pseudomonas_Other & 2.38 & PSO_L & 2.00 & 0.008 \\
\hline \multicolumn{5}{|l|}{ Axilla } \\
\hline Selenomonas_s_noxia & 1.25 & Healthy & 2.13 & 0.028 \\
\hline Paracoccus_s_aminovorans & 2.61 & Healthy & 2.37 & 0.006 \\
\hline Lactobacillus_s__ & 3.19 & Healthy & 2.94 & 0.019 \\
\hline Propionibacterium_Other & 1.33 & PSO_L & 2.09 & 0.041 \\
\hline Bradyrhizobium_s__ & 1.55 & PSO_N & 2.16 & 0.048 \\
\hline Methylopila_s__ & 2.33 & PSO_N & 2.13 & 0.048 \\
\hline Veillonella_s_dispar & 2.71 & PSO_N & 2.44 & 0.013 \\
\hline Peptostreptococcus_s__ & 1.50 & PSO_N & 2.15 & 0.048 \\
\hline Rhodococcus_s__ & 2.28 & PSO_N & 2.09 & 0.015 \\
\hline Streptococcus_s__ & 3.84 & PSO_N & 3.48 & 0.021 \\
\hline \multicolumn{5}{|l|}{ Gluteal fold } \\
\hline Propionibacterium_s_acnes & 5.38 & Healthy & 4.78 & 0.043 \\
\hline Mycobacterium_Other & 3.65 & Healthy & 3.33 & 0.002 \\
\hline Propionibacterium_s_granulosum & 3.59 & Healthy & 3.07 & 0.031 \\
\hline Mitsuokella_s__ & 2.64 & Healthy & 2.55 & 0.012 \\
\hline Amaricoccus_s__ & 2.73 & Healthy & 2.42 & 0.043 \\
\hline Mycobacterium_s_vaccae & 2.04 & Healthy & 2.12 & 0.018 \\
\hline \multicolumn{5}{|l|}{ Scalp } \\
\hline Flavobacterium_s__ & 2.58 & PSO_L & 2.17 & 0.001 \\
\hline Pseudomonas_s_fragi & 3.09 & PSO_L & 2.70 & 0.011 \\
\hline Pseudomonas_s__ & 2.73 & PSO_N & 2.22 & 0.025 \\
\hline Sphingomonas_s__ & 3.15 & PSO_N & 2.78 & 0.006 \\
\hline Staphylococcus_Other & 3.41 & PSO_N & 2.87 & 0.040 \\
\hline
\end{tabular}


Table 9 Sample information of S. aureus high samples

\begin{tabular}{|c|c|c|c|c|c|c|}
\hline \#PID & Skin_type & Skin_site & Disease state & S. aureus_abundance & Baseline & Fold change \\
\hline 7319 & Sebaceous & Scalp & PSO_L & 0.6119 & 0.0068 & 90.0 \\
\hline 7314 & Dry & Leg & PSO_L & 0.4699 & 0.0068 & 69.1 \\
\hline 7319 & Sebaceous & Scalp & PSO_N & 0.2857 & 0.0068 & 42.0 \\
\hline 7319 & Dry & Trunk & PSO_N & 0.1300 & 0.0068 & 19.1 \\
\hline 7306 & Sebaceous & Scalp & PSO_L & 0.1085 & 0.0068 & 16.0 \\
\hline 7331 & Sebaceous & Scalp & PSO_L & 0.0863 & 0.0068 & 12.7 \\
\hline 7331 & Dry & Arm & PSO_L & 0.0760 & 0.0068 & 11.2 \\
\hline 7313 & Sebaceous & Scalp & PSO_N & 0.0647 & 0.0068 & 9.5 \\
\hline 7319 & Dry & Arm & PSO_L & 0.0569 & 0.0068 & 8.4 \\
\hline 7331 & Dry & Leg & PSO_L & 0.0559 & 0.0068 & 8.2 \\
\hline 7319 & Dry & Arm & PSO_N & 0.0454 & 0.0068 & 6.7 \\
\hline 7331 & Dry & Arm & PSO_N & 0.0424 & 0.0068 & 6.2 \\
\hline 7331 & Sebaceous & Scalp & PSO_N & 0.0378 & 0.0068 & 5.6 \\
\hline 7331 & Dry & Leg & PSO_N & 0.0344 & 0.0068 & 5.1 \\
\hline 7331 & Moist & Axilla & PSO_N & 0.0295 & 0.0068 & 4.3 \\
\hline 7331 & Moist & Axilla & PSO_L & 0.0288 & 0.0068 & 4.2 \\
\hline 7319 & Moist & Gluteal_fold & PSO_N & 0.0260 & 0.0068 & 3.8 \\
\hline 7331 & Dry & Trunk & PSO_N & 0.0219 & 0.0068 & 3.2 \\
\hline 7331 & Dry & Trunk & PSO_L & 0.0201 & 0.0068 & 3.0 \\
\hline 7319 & Dry & Leg & PSO_L & 0.0157 & 0.0068 & 2.3 \\
\hline 7306 & Dry & Arm & PSO_L & 0.0120 & 0.0068 & 1.8 \\
\hline 7306 & Dry & Trunk & PSO_L & 0.0118 & 0.0068 & 1.7 \\
\hline 7302 & Sebaceous & Scalp & PSO_L & 0.0116 & 0.0068 & 1.7 \\
\hline 7327 & Dry & Leg & PSO_L & 0.0106 & 0.0068 & 1.6 \\
\hline 7319 & Dry & Leg & PSO_N & 0.0104 & 0.0068 & 1.5 \\
\hline 7306 & Moist & Gluteal_fold & PSO_N & 0.0101 & 0.0068 & 1.5 \\
\hline 7331 & Moist & Gluteal_fold & PSO_N & 0.0100 & 0.0068 & 1.5 \\
\hline 7314 & Moist & Gluteal_fold & PSO_L & 0.0089 & 0.0068 & 1.3 \\
\hline 7305 & Dry & Arm & PSO_N & 0.0085 & 0.0068 & 1.3 \\
\hline 7306 & Dry & Leg & PSO_L & 0.0080 & 0.0068 & 1.2 \\
\hline 7306 & Dry & Leg & PSO_N & 0.0078 & 0.0068 & 1.1 \\
\hline 7309 & Dry & Arm & PSO_N & 0.0070 & 0.0068 & 1.0 \\
\hline
\end{tabular}

indicates that the association of S. aureus with psoriasis is not driven by any single anatomic site and that the presence of abundant $S$. aureus in only a subset of psoriasis patients, at different anatomic locations, results in an underpowered sample size for detection of S. aureus at any single body site.

\section{Correlations between different bacterial species}

Like any ecosystem, the composition of skin microbiome is modulated by both environmental factors (i.e., nutrient availability and host immune response) and interactions between different bacterial species. Inter-microbial interactions can be a major driver of microbial community composition, and understanding this interaction can yield important insights regarding the establishment and maintenance of psoriasis-associated microbial communities. We further investigated this microbe-microbe interaction by correlating microbial abundances with each other. At the genus level, we identified three clusters of bacterial communities, each constituting a group of bacteria significantly correlated in abundance (Fig. 4a). Cluster A was the largest cluster and consisted of Corynebacterium, Porphyromonas, Prevotella, Peptoniphilus, Finegoldia, and Anaercoccus. Cluster B was composed of Kocuria, Paracoccus, Micrococcus, and Janibacter. Lastly, Cluster C consisted of strongly correlated Streptococcus and Rothia. Given the 


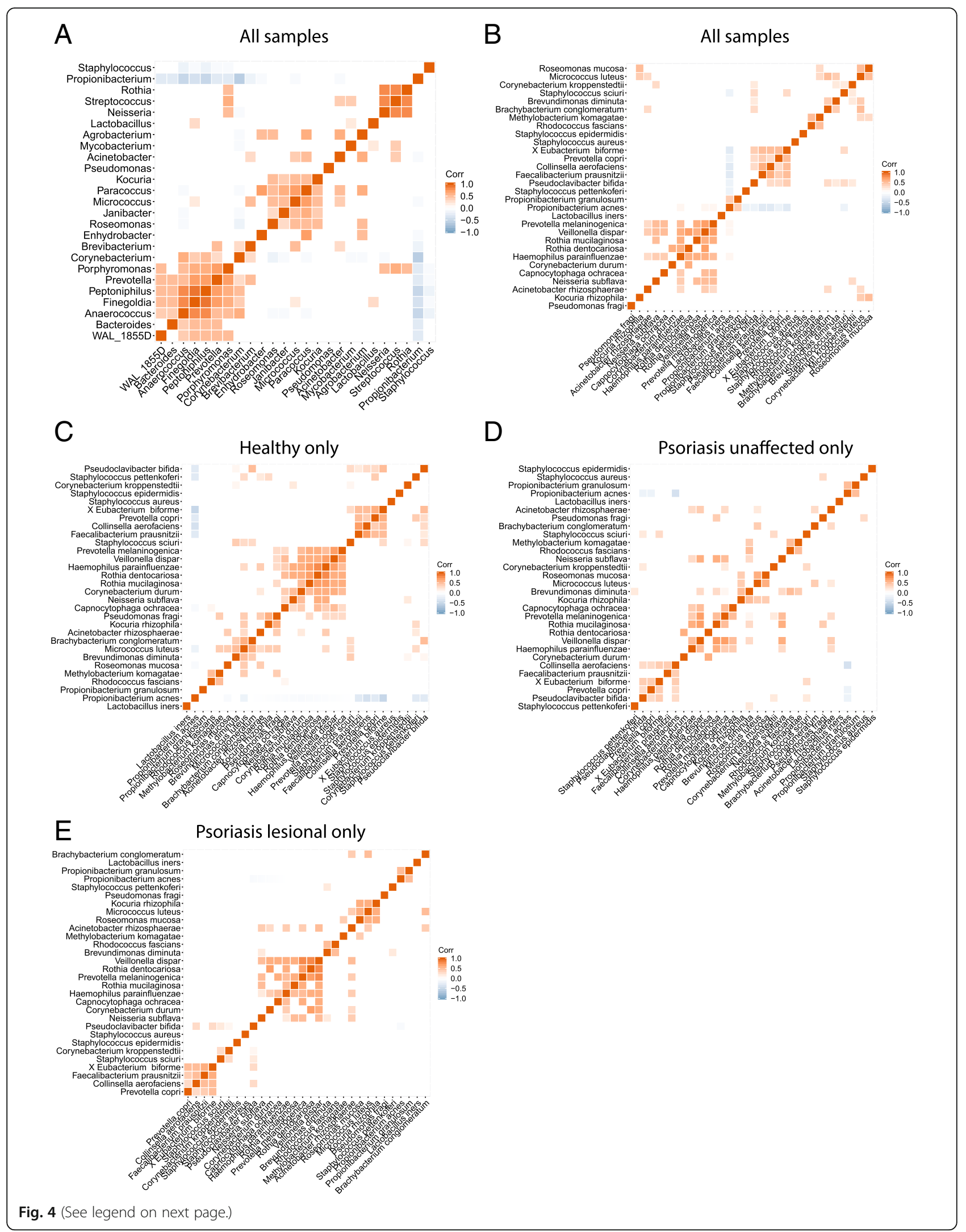


(See figure on previous page.)

Fig. 4 Correlations between the most abundant bacterial genera and species. Correlation plots show the Spearman correlations among a the top 25 most abundant genera or $\mathbf{b}$ the top 30 most abundant species in all samples. Correlations among the top 30 most abundant bacterial species associated with $\mathbf{c}$ Healthy skin samples, $\mathbf{d}$ psoriasis unaffected samples, and e psoriasis lesional samples. Only the correlations with statistical significance are shown. Color and intensity indicate directions and strength of the correlation

previous reports of the potential role of Streptococcus in driving psoriasis $[9,10]$, it would be interesting to further investigate the role of Rothia spp. in psoriasis since it is highly co-abundant with Streptococcus. At the species level, P. acnes, which was more abundant in healthy skin, was negatively correlated with $S$. sciuri and S. pettenkoferi, both of which were enriched in the skin microbiota of psoriasis patients (Fig. 4b). Consistent with this observation, we also found P. acnes and S. epidermidis to be significantly enriched in $S$. aureus low psoriasis samples and $S$. pettenkoferi was enriched in S. aureus high psoriasis samples (Additional file 1: Figure S3E), suggesting that the antagonistic interaction among these bacteria may contribute to pathogenesis. Interestingly, Pseudoclavibacter bifida was negatively correlated with $P$. acnes and positively correlated to S. sciuri (Fig. 4b). The abundance of Pseudoclavibacter bifida was also enriched in S. aureus high psoriasis samples (Additional file 1: Figure S3E). Moreover, $P$. acnes and $P$. granulosum serve as two predominant Propionibacterium species and our data shows that they are positively correlated with each other (Fig. 4b). The strong co-correlation of $P$. acnes and P. granulosum and their association to healthy skin suggests that these Propionibacterium spp. may have a role in maintaining skin health.

Psoriatic lesions are characterized by thick and highly inflamed skin plaques, so the psoriatic lesions, psoriasis non-lesional skin, and healthy skin represent very distinct microbial habitats that may affect the quality of interactions between different microbes. Consistent with this, we observed distinct species correlations in these disease states, supporting the hypothesis that different microbe-microbe interactions occur in each disease state. We found the most numerous and strongest microbe-microbe correlations in healthy skin samples (Fig. 4c). Surprisingly, species correlations in the microbial community associated with psoriatic lesions (Fig. 4e) were more similar to those in healthy skin than psoriatic non-lesional skin (Fig. 4d). In healthy skin, P. acnes was negatively correlated with several bacterial species (Fig. 4c), suggesting it may inhibit the growth of these bacteria. Fewer microbes were negatively correlated with $P$. acnes in psoriatic non-lesional skin (Fig. 4d) and only Pseudoclavibacter bifida was anti-correlated with $P$. acnes in lesional skin (Fig. 4e). Overall, our data suggests the possibility that $P$. acnes may have a role in influencing the skin microbial community by keeping the growth of some microbes under control and that perturbation of this balance in psoriatic skin could serve as a potential disease driver.

\section{S. aureus triggers Th17 immune response in a murine model}

The increased prevalence of $S$. aureus in both lesional and non-lesional skin of psoriasis patients suggested the possibility that $S$. aureus might play a role in early stages of psoriasis pathogenesis. Despite its undesirable role in the context of psoriasis, the Th17 response serves as one of the major arms of host defense against bacterial infection through promotion of $\mathrm{B}$ cell activation and attraction of neutrophils [36, 37]. IL-17 is crucial in clearance of $S$. aureus at nasal, skin, and soft tissue sites [37]. Previous studies have shown that $S$. aureus proteins promote Th17 differentiation in vitro [38], suggesting that colonization by $S$. aureus can lead to increased Th17 activation and IL-17 secretion. To assess the effect of $S$. aureus colonization on Th17 response in the skin, we performed skin colonization of newborn-specific pathogen-free (SPF) mice with $S$. aureus strain USA300 and assessed the cutaneous effector $\mathrm{CD}_{4}^{+} \mathrm{T}$ (Teff) cell response using RNAseq in comparison with SPF mice colonized with the commensal S. epidermidis, or un-colonized SPF controls (Table 10). We found significantly stronger Th17 transcriptomic signals in Teff cells sorted from the skin S. aureus-colonized mice. Teff cells isolated from mice exposed to $S$. aureus expressed significantly higher levels of IL-17A and IL-17F cytokine transcripts (Fig. 5a, b). IL-17A has been well characterized as one of the major drivers for psoriasis pathogenesis whereas IL-17F shares some redundant functions to IL-17A but its role in psoriasis is less defined [39]. Besides IL-17, other components of Th17 responses including IL23R and IL22 were also increased upon $S$. aureus exposure (Fig. 5c, d). While $S$. aureus exposure during early life triggers a strong Th17 response in mice, the same treatment did not elicit consistent activation of a Th1 response (Additional file 1: Figure S4). Although S. aureus colonization has been strongly associated with atopic dermatitis, which is driven by Th2 responses $[8,40,41]$, most components of the Th2 response such as IL-4, IL-5, and IL-13 were not induced by early colonization of $S$. aureus (Additional file 1: Figure S5). However, we did observe a strong induction in expression of the Th2-promoting transcription factor, GATA3 (Additional file 1: Figure S5E). Together, our data suggests that $S$. aureus colonization can specifically trigger 
Table 10 Genes differentially expressed in skin T effector cells of SPF + SA-colonized mice vs. SPF-colonized mice

\begin{tabular}{|c|c|c|c|c|c|c|c|c|c|}
\hline Gene & Log2FoldChange & $p$ value & $p$ adj & $\mathrm{absFC}$ & Gene & Log2FoldChange & $p$ value & $p$ adj & absFC \\
\hline Adgrl4 & 12.05 & 1.11E-21 & $7.51 \mathrm{E}-19$ & 12.05 & Alas2 & 10.11 & $4.62 \mathrm{E}-13$ & $5.13 \mathrm{E}-11$ & 10.11 \\
\hline Efnb2 & 12.04 & 1.30E-20 & $7.82 \mathrm{E}-18$ & 12.04 & Aadac & 10.10 & 7.90E-08 & $2.09 \mathrm{E}-06$ & 10.10 \\
\hline Ptprb & 11.95 & $2.57 \mathrm{E}-16$ & $5.87 \mathrm{E}-14$ & 11.95 & Unc45b & 10.10 & $3.29 \mathrm{E}-11$ & 2.14E-09 & 10.10 \\
\hline Sele & 11.47 & $6.20 \mathrm{E}-10$ & $2.94 \mathrm{E}-08$ & 11.47 & Enpp4 & 10.07 & $5.32 \mathrm{E}-13$ & $5.72 \mathrm{E}-11$ & 10.07 \\
\hline Procr & 11.41 & 7.75E-12 & $6.08 \mathrm{E}-10$ & 11.41 & Рappa2 & 10.06 & $4.75 \mathrm{E}-12$ & 4.05E-10 & 10.06 \\
\hline$\| 17 a$ & 11.38 & $5.36 \mathrm{E}-27$ & $1.29 \mathrm{E}-23$ & 11.38 & Tcrg-C4 & 10.04 & $6.93 \mathrm{E}-14$ & $9.59 \mathrm{E}-12$ & 10.04 \\
\hline Galnt15 & 11.37 & 7.57E-20 & $3.87 \mathrm{E}-17$ & 11.37 & Bcam & 10.02 & $2.25 \mathrm{E}-13$ & $2.67 \mathrm{E}-11$ & 10.02 \\
\hline Cyyr1 & 11.28 & $2.11 \mathrm{E}-18$ & $7.90 \mathrm{E}-16$ & 11.28 & Tie1 & 10.01 & 7.30E-12 & $5.78 \mathrm{E}-10$ & 10.01 \\
\hline Rgs4 & 11.28 & $2.77 \mathrm{E}-20$ & $1.51 \mathrm{E}-17$ & 11.28 & Gja5 & 9.99 & $2.45 \mathrm{E}-10$ & $1.28 \mathrm{E}-08$ & 9.99 \\
\hline Flt1 & 11.27 & $4.15 \mathrm{E}-17$ & $1.17 \mathrm{E}-14$ & 11.27 & Rassf9 & 9.98 & $1.20 \mathrm{E}-14$ & 1.97E-12 & 9.98 \\
\hline Btnl9 & 11.24 & $1.83 \mathrm{E}-17$ & $5.72 \mathrm{E}-15$ & 11.24 & Tfap2c & 9.98 & $6.73 \mathrm{E}-16$ & $1.37 \mathrm{E}-13$ & 9.98 \\
\hline Nts & 11.24 & $1.35 \mathrm{E}-13$ & $1.72 \mathrm{E}-11$ & 11.24 & Gdap10 & 9.97 & 1.67E-16 & 4.16E-14 & 9.97 \\
\hline Lamb2 & 11.07 & $2.31 \mathrm{E}-16$ & $5.41 \mathrm{E}-14$ & 11.07 & Ppm1l & 9.93 & $7.46 \mathrm{E}-14$ & $1.02 \mathrm{E}-11$ & 9.93 \\
\hline Car4 & 11.00 & 1.67E-18 & $6.41 \mathrm{E}-16$ & 11.00 & Ptch2 & 9.92 & $1.15 \mathrm{E}-12$ & $1.15 E-10$ & 9.92 \\
\hline LsS & 10.97 & $5.12 \mathrm{E}-12$ & $4.30 \mathrm{E}-10$ & 10.97 & $V \operatorname{tn}$ & 9.91 & $6.14 \mathrm{E}-12$ & $5.01 \mathrm{E}-10$ & 9.91 \\
\hline Blk & 10.97 & 1.17E-09 & $5.16 \mathrm{E}-08$ & 10.97 & DII4 & 9.89 & $3.19 \mathrm{E}-14$ & 4.67E-12 & 9.89 \\
\hline RP24-360B3.1 & 10.84 & 1.60E-16 & $4.02 \mathrm{E}-14$ & 10.84 & Fam73a & 9.82 & 1.17E-13 & $1.53 \mathrm{E}-11$ & 9.82 \\
\hline Tinagl1 & 10.84 & 4.26E-19 & $1.85 \mathrm{E}-16$ & 10.84 & Pcdh17 & 9.82 & $2.72 \mathrm{E}-14$ & $4.10 E-12$ & 9.82 \\
\hline Stc1 & 10.80 & $1.80 \mathrm{E}-12$ & $1.69 \mathrm{E}-10$ & 10.80 & Ptgs1 & 9.80 & $8.06 \mathrm{E}-12$ & $6.24 E-10$ & 9.80 \\
\hline Me1 & 10.78 & $2.90 \mathrm{E}-15$ & $5.20 \mathrm{E}-13$ & 10.78 & Slc12a1 & 9.79 & $1.88 \mathrm{E}-14$ & $2.96 \mathrm{E}-12$ & 9.79 \\
\hline Aqp7 & 10.73 & $2.58 \mathrm{E}-12$ & $2.27 \mathrm{E}-10$ & 10.73 & Gm37297 & 9.77 & $4.01 \mathrm{E}-16$ & $8.79 E-14$ & 9.77 \\
\hline Ptprm & 10.69 & $1.62 \mathrm{E}-17$ & $5.16 \mathrm{E}-15$ & 10.69 & Cypla1 & 9.74 & $5.32 \mathrm{E}-08$ & 1.49E-06 & 9.74 \\
\hline Rasip1 & 10.62 & 1.87E-12 & $1.73 \mathrm{E}-10$ & 10.62 & Shroom4 & 9.73 & 1.35E-09 & $5.84 \mathrm{E}-08$ & 9.73 \\
\hline Itga7 & 10.61 & $4.83 \mathrm{E}-15$ & $8.40 \mathrm{E}-13$ & 10.61 & Gm12158 & 9.72 & $1.18 \mathrm{E}-13$ & $1.53 \mathrm{E}-11$ & 9.72 \\
\hline Rbp7 & 10.56 & $5.20 \mathrm{E}-09$ & $1.99 \mathrm{E}-07$ & 10.56 & Ackr3 & 9.71 & $2.01 \mathrm{E}-16$ & $4.86 \mathrm{E}-14$ & 9.71 \\
\hline Selp & 10.54 & $7.89 \mathrm{E}-12$ & $6.13 \mathrm{E}-10$ & 10.54 & Clstn3 & 9.67 & 7.71E-11 & 4.57E-09 & 9.67 \\
\hline$\| 17 f$ & 10.53 & $2.25 \mathrm{E}-14$ & $3.42 \mathrm{E}-12$ & 10.53 & Hmcn1 & 9.64 & $6.27 \mathrm{E}-16$ & $1.29 E-13$ & 9.64 \\
\hline Angpt2 & 10.50 & $2.37 \mathrm{E}-12$ & $2.14 \mathrm{E}-10$ & 10.50 & Tek & 9.63 & 3.10E-19 & $1.46 \mathrm{E}-16$ & 9.63 \\
\hline Hid1 & 10.47 & $3.39 \mathrm{E}-17$ & $1.00 \mathrm{E}-14$ & 10.47 & Esrp2 & 9.62 & $6.62 \mathrm{E}-11$ & $3.98 \mathrm{E}-09$ & 9.62 \\
\hline Adamts9 & 10.45 & 7.13E-16 & $1.43 \mathrm{E}-13$ & 10.45 & Ednra & 9.61 & 4.06E-08 & $1.18 \mathrm{E}-06$ & 9.61 \\
\hline Sqle & 10.44 & $2.08 \mathrm{E}-13$ & $2.51 \mathrm{E}-11$ & 10.44 & Thsd7a & 9.60 & $3.99 \mathrm{E}-10$ & $2.01 \mathrm{E}-08$ & 9.60 \\
\hline Pdgfb & 10.44 & $7.58 \mathrm{E}-12$ & $5.98 \mathrm{E}-10$ & 10.44 & Tdrd9 & 9.59 & $1.23 \mathrm{E}-08$ & 4.19E-07 & 9.59 \\
\hline Gpihbp1 & 10.41 & 1.07E-16 & $2.77 \mathrm{E}-14$ & 10.41 & Sfrp1 & 9.56 & $1.80 \mathrm{E}-10$ & $9.60 \mathrm{E}-09$ & 9.56 \\
\hline Galnt18 & 10.37 & $4.46 \mathrm{E}-17$ & $1.23 \mathrm{E}-14$ & 10.37 & Aplnr & 9.56 & $1.46 \mathrm{E}-17$ & $4.83 \mathrm{E}-15$ & 9.56 \\
\hline Sned1 & 10.36 & $3.86 \mathrm{E}-13$ & $4.37 \mathrm{E}-11$ & 10.36 & Mall & 9.55 & $1.20 \mathrm{E}-14$ & $1.97 \mathrm{E}-12$ & 9.55 \\
\hline Sulf1 & 10.32 & $6.17 \mathrm{E}-16$ & $1.29 \mathrm{E}-13$ & 10.32 & $M \times 2$ & 9.55 & $2.10 \mathrm{E}-13$ & $2.51 \mathrm{E}-11$ & 9.55 \\
\hline Lamc3 & 10.30 & $1.44 \mathrm{E}-10$ & $7.91 \mathrm{E}-09$ & 10.30 & Snca & 9.55 & $7.99 \mathrm{E}-08$ & $2.11 \mathrm{E}-06$ & 9.55 \\
\hline Stap2 & 10.25 & $8.80 \mathrm{E}-19$ & $3.62 E-16$ & 10.25 & Gm15740 & 9.55 & 4.67E-14 & $6.73 E-12$ & 9.55 \\
\hline Ccm2l & 10.22 & $2.11 \mathrm{E}-14$ & $3.27 \mathrm{E}-12$ & 10.22 & Sectm1b & 9.54 & $6.10 \mathrm{E}-15$ & $1.05 E-12$ & 9.54 \\
\hline Tnc & 10.21 & $2.22 \mathrm{E}-12$ & $2.03 E-10$ & 10.21 & Tacr1 & 9.53 & $1.24 \mathrm{E}-07$ & $3.06 \mathrm{E}-06$ & 9.53 \\
\hline Esrp1 & 10.17 & $7.74 \mathrm{E}-10$ & $3.56 \mathrm{E}-08$ & 10.17 & Rapgef5 & 9.52 & $1.49 \mathrm{E}-14$ & $2.42 \mathrm{E}-12$ & 9.52 \\
\hline Cyp17a1 & 10.15 & 2.27E-08 & 7.07E-07 & 10.15 & Clec1a & 9.52 & $5.45 \mathrm{E}-07$ & $1.10 \mathrm{E}-05$ & 9.52 \\
\hline Nrip2 & 10.15 & $1.06 \mathrm{E}-16$ & 2.77E-14 & 10.15 & Prox1 & 9.51 & 1.31E-09 & 5.71E-08 & 9.51 \\
\hline
\end{tabular}

Table 10 Genes differentially expressed in skin T effector cells of SPF + SA-colonized mice vs. SPF-colonized mice (Continued)

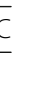


Table 10 Genes differentially expressed in skin T effector cells of SPF + SA-colonized mice vs. SPF-colonized mice (Continued)

\begin{tabular}{lllll}
\hline Gene & Log2FoldChange & $p$ value & $p$ adj & absFC \\
\hline Clec14a & 9.51 & $7.87 \mathrm{E}-08$ & $2.09 \mathrm{E}-06$ & 9.51
\end{tabular}

Rasgrf2

DII1

9.50

Stac2

9.50

Celsr2

$$
9.50
$$

Robo1

$$
9.49
$$

Cxadr

$$
9.48
$$

Clic5

$$
9.47
$$

Taf9b

$$
9.46
$$

Spns2

9.46

Dhcr24

9.46

Gm37736

9.45

Gm16587

9.45

Ret

Tmem45b

1|22

Upp1

Paqr5

Piezo2

Psd2

Adtrp

Gk5

Cldn15

Aqp1

Heph

Emilin1

Plxna4

Ano1

Ebf3

Sgip1

Gm38125

Avpr1a

Tcrg-V6

Hoxd10

9.33

Dchs

9.30

Hrct1

9.30

Clqtnf9

9.29

Lrg1

9.28

Fgfbp1 $\quad 9.24$

Eps8l2 $\quad 9.24$

RP24-188E19.4 9.22

Vsig10 9.21

Exd2

9.21

$5.56 \mathrm{E}-12 \quad 4.62 \mathrm{E}-10 \quad 9.50$

$1.93 \mathrm{E}-15 \quad 3.62 \mathrm{E}-13 \quad 9.50$

8.92E-12 6.70E-10 9.50

$1.88 \mathrm{E}-11 \quad 1.27 \mathrm{E}-09 \quad 9.49$

1.30E-10 7.27E-09 9.48

$7.82 \mathrm{E}-12 \quad 6.11 \mathrm{E}-10 \quad 9.47$

7.72E-09 2.82E-07 9.46

4.11E-14 5.98E-12 9.46

$2.74 \mathrm{E}-12 \quad 2.38 \mathrm{E}-10 \quad 9.46$

$2.55 \mathrm{E}-13 \quad 2.94 \mathrm{E}-11 \quad 9.45$

$2.18 \mathrm{E}-14 \quad 3.34 \mathrm{E}-12 \quad 9.45$

5.16E-07 1.05E-05 9.43

2.75E-08 8.36E-07 9.43

1.37E-06 2.44E-05 9.43

$1.82 \mathrm{E}-12 \quad 1.70 \mathrm{E}-10 \quad 9.42$

$1.49 \mathrm{E}-13 \quad 1.88 \mathrm{E}-11 \quad 9.41$

8.56E-10 3.84E-08 9.41

$1.23 \mathrm{E}-10 \quad 6.96 \mathrm{E}-09 \quad 9.41$

$5.60 \mathrm{E}-10 \quad 2.76 \mathrm{E}-08 \quad 9.40$

4.97E-08 1.41E-06 9.40

6.11E-11 3.73E-09 9.39

$1.51 \mathrm{E}-13 \quad 1.90 \mathrm{E}-11 \quad 9.39$

$7.85 E-13 \quad 8.13 E-11 \quad 9.38$

$1.81 \mathrm{E}-15 \quad 3.44 \mathrm{E}-13 \quad 9.37$

2.32E-09 9.56E-08 9.36

7.20E-09 2.67E-07 9.36

$2.28 \mathrm{E}-12 \quad 2.07 \mathrm{E}-10 \quad 9.36$

$3.58 \mathrm{E}-10 \quad 1.82 \mathrm{E}-08 \quad 9.35$

4.44E-11 2.83E-09 9.34

9.93E-08 2.55E-06 9.34

7.86E-10 3.57E-08 9.34

$5.26 \mathrm{E}-14 \quad 7.46 \mathrm{E}-12 \quad 9.33$

$1.14 \mathrm{E}-13 \quad 1.51 \mathrm{E}-11 \quad 9.30$

$1.07 \mathrm{E}-08 \quad 3.74 \mathrm{E}-07 \quad 9.30$

3.45E-17 1.00E-14 9.29

$1.31 \mathrm{E}-15 \quad 2.55 \mathrm{E}-13 \quad 9.28$

$1.99 \mathrm{E}-07 \quad 4.60 \mathrm{E}-06 \quad 9.24$

5.95E-12 4.87E-10 9.24

6.55E-11 3.95E-09 9.22

4.56E-09 1.76E-07 9.21

$2.86 \mathrm{E}-14 \quad 4.28 \mathrm{E}-12 \quad 9.21$
1.43E-07 3.46E-06 9.40
Table 10 Genes differentially expressed in skin T effector cells of SPF + SA-colonized mice vs. SPF-colonized mice (Continued)

\begin{tabular}{lllll}
\hline Gene & Log2FoldChange & $p$ value & $p$ adj & absFC \\
\hline Rnd1 & 9.20 & $2.14 \mathrm{E}-10$ & $1.13 \mathrm{E}-08$ & 9.20
\end{tabular}

Adgrg6 $\quad 9.19$

4.55E-11 2.88E-09 9.19

Adcy4 $\quad 9.18$

1.40E-10 7.73E-09 9.18

Cyp2b19 $\quad 9.18$

Ndst3 $\quad 9.15$

$\mathrm{XIr4C \quad 9.14}$

RP24-188E19.3 9.14

Stc2 9.14

Lmbr1

9.13

2610012C04Rik 9.13

Yes1

9.12

Sgcb

9.11

Vsig2

9.11

Zfpm2

9.10

9.10

Oaf

9.09

Sybu

9.08

Ebf2

9.08

Gm26667

9.08

9.07

9.07

9.06

9.05

Grrp1

9.04

Slc2a13

9.02

$\begin{array}{ll}\text { Gm37783 } & 9.02 \\ \text { Fat4 } & 9.01\end{array}$

Slc33a1 $\quad 9.00$

Gtf2h2 9.00

C130079G13Rik 9.00

Col6a6 $\quad 9.00$

Adamtsl1 $\quad 8.99$

Pcdh12 8.98

Npy1r

8.98

8.96

Tfap2a

8.95

Gm38157

8.95

Adgrf5 $\quad 8.95$

Sumf2 8.95

Moxd1 8.93

Fdxr $\quad 8.93$

Colec11

8.92

St6galnac5 8.92

Pparg

8.91
2.24E-07 5.05E-06 9.18

$1.94 \mathrm{E}-09 \quad 8.13 \mathrm{E}-08 \quad 9.15$

$9.66 \mathrm{E}-13 \quad 9.82 \mathrm{E}-11 \quad 9.14$

$6.41 \mathrm{E}-13 \quad 6.76 \mathrm{E}-11 \quad 9.14$

2.37E-08 7.35E-07 9.14

2.35E-07 5.24E-06 9.13

3.39E-11 2.19E-09 9.13

$9.29 \mathrm{E}-09 \quad 3.32 \mathrm{E}-07 \quad 9.12$

4.81E-08 1.37E-06 9.11

7.85E-08 2.09E-06 9.11

$9.96 \mathrm{E}-12 \quad 7.37 \mathrm{E}-10 \quad 9.10$

1.97E-07 4.55E-06 9.10

1.23E-09 5.41E-08 9.09

2.05E-14 3.20E-12 9.08

$1.06 \mathrm{E}-13 \quad 1.42 \mathrm{E}-11 \quad 9.08$

4.10E-08 1.19E-06 9.08

9.53E-11 5.53E-09 9.07

4.08E-09 1.59E-07 9.07

8.47E-12 6.47E-10 9.06

2.00E-11 1.34E-09 9.05

5.70E-12 4.69E-10 9.04

$1.95 E-13 \quad 2.39 E-11 \quad 9.02$

$1.54 \mathrm{E}-09 \quad 6.59 \mathrm{E}-08 \quad 9.01$

1.77E-11 1.20E-09 9.00

$1.32 \mathrm{E}-10 \quad 7.39 \mathrm{E}-09 \quad 9.00$

5.71E-06 $\quad 8.18 \mathrm{E}-05 \quad 9.00$

$2.41 \mathrm{E}-12 \quad 2.16 \mathrm{E}-10 \quad 9.00$

6.25E-07 1.23E-05 8.99

$2.24 \mathrm{E}-08 \quad 6.99 \mathrm{E}-07 \quad 8.98$

$1.53 \mathrm{E}-11 \quad 1.06 \mathrm{E}-09 \quad 8.98$

1.39E-06 2.47E-05 8.96

7.45E-10 3.47E-08 8.95

4.42E-07 9.16E-06 8.95

4.35E-24 4.89E-21 8.95

4.48E-08 1.28E-06 8.95

$1.56 \mathrm{E}-10 \quad 8.50 \mathrm{E}-09 \quad 8.93$

3.58E-09 1.41E-07 8.93

6.02E-10 2.87E-08 8.92

6.51E-10 3.06E-08 8.92

$3.72 \mathrm{E}-08 \quad 1.10 \mathrm{E}-06 \quad 8.91$ 
Table 10 Genes differentially expressed in skin T effector cells of SPF + SA-colonized mice vs. SPF-colonized mice (Continued)

\begin{tabular}{lllll}
\hline Gene & Log2FoldChange & $p$ value & $p$ adj & absFC \\
\hline Ndnf & 8.90 & $5.09 E-10$ & $2.53 \mathrm{E}-08$ & 8.90
\end{tabular}

Gm15712

Tcaf2

8.90

8.90

Adam12

8.90

Vstm4

8.89

Pkn3

8.88

RP23-363 M4.2 8.86

Neurl1b

8.84

4631405K08Rik 8.84

Dennd2C 8.83

II23r $\quad 8.82$

Cda $\quad 8.82$

Higd1b $\quad 8.81$

Plscr2 8.81

Len2 8.80

Lrrn1 $\quad 8.80$

Nipsnap1 $\quad 8.78$

Zfp57 8.77

Reep6 $\quad 8.77$

Dach1 8.76

Cpa6 $\quad 8.76$

Scube1 $\quad 8.76$

Tmem51 8.75

Prlr $\quad 8.74$

Nova2 $\quad 8.74$

Arnt2 8.73

Cldn5 $\quad 8.73$

Slc6a17 $\quad 8.73$

Gucy1b3 $\quad 8.73$

Nrbp2 8.73

Fgfrl1 $\quad 8.72$

Fam13c $\quad 8.72$

RP24-9504.6 8.71

Apmap $\quad 8.71$

Crygd $\quad-8.70$

Gdf10 $\quad 8.70$

Plxnb1 $\quad 8.68$

Foxc1 8.67

Has1 $\quad 8.67$

Hs3st1 8.67

Ppp1r3c $\quad 8.66$

$\mathrm{Dio2} \quad 8.66$

Gjc3

8.66

$3.20 \mathrm{E}-12 \quad 2.77 \mathrm{E}-10 \quad 8.90$

7.47E-08 2.00E-06 8.90

$1.98 \mathrm{E}-07 \quad 4.57 \mathrm{E}-06 \quad 8.90$

$1.24 \mathrm{E}-06 \quad 2.23 \mathrm{E}-05 \quad 8.89$

$2.55 \mathrm{E}-08 \quad 7.88 \mathrm{E}-07 \quad 8.88$

8.36E-12 6.41E-10 8.86

5.73E-07 1.14E-05 8.84

$6.73 \mathrm{E}-13 \quad 7.01 \mathrm{E}-11 \quad 8.84$

1.57E-08 5.17E-07 8.83

$3.27 \mathrm{E}-12 \quad 2.82 \mathrm{E}-10 \quad 8.82$

$1.11 \mathrm{E}-08 \quad 3.84 \mathrm{E}-07 \quad 8.82$

2.23E-06 3.63E-05 8.81

$1.11 \mathrm{E}-06 \quad 2.03 \mathrm{E}-05 \quad 8.81$

4.39E-10 2.21E-08 8.80

$5.24 \mathrm{E}-08 \quad 1.48 \mathrm{E}-06 \quad 8.80$

3.79E-06 5.75E-05 8.78

$9.44 \mathrm{E}-13 \quad 9.65 \mathrm{E}-11 \quad 8.77$

$2.48 \mathrm{E}-07 \quad 5.51 \mathrm{E}-06 \quad 8.77$

5.34E-07 1.08E-05 8.76

$1.47 \mathrm{E}-11 \quad 1.04 \mathrm{E}-09 \quad 8.76$

$1.49 \mathrm{E}-07 \quad 3.58 \mathrm{E}-06 \quad 8.76$

5.87E-10 2.82E-08 8.75

$5.71 \mathrm{E}-11 \quad 3.52 \mathrm{E}-09 \quad 8.74$

4.46E-07 9.23E-06 8.74

$1.01 \mathrm{E}-07 \quad 2.59 \mathrm{E}-06 \quad 8.73$

$8.94 \mathrm{E}-12 \quad 6.70 \mathrm{E}-10 \quad 8.73$

4.66E-09 1.79E-07 8.73

$1.90 \mathrm{E}-07 \quad 4.42 \mathrm{E}-06 \quad 8.73$

5.74E-06 8.20E-05 8.73

5.81E-10 2.80E-08 8.72

$2.88 \mathrm{E}-06 \quad 4.53 \mathrm{E}-05 \quad 8.72$

2.13E-06 3.50E-05 8.71

$9.28 \mathrm{E}-09 \quad 3.32 \mathrm{E}-07 \quad 8.71$

3.17E-04 2.36E-03 8.70

$1.43 \mathrm{E}-08 \quad 4.75 \mathrm{E}-07 \quad 8.70$

$4.34 \mathrm{E}-08 \quad 1.25 \mathrm{E}-06 \quad 8.68$

3.07E-11 2.00E-09 8.67

3.76E-19 1.67E-16 8.67

8.06E-07 1.53E-05 8.67

$1.84 \mathrm{E}-07 \quad 4.33 \mathrm{E}-06 \quad 8.66$

7.06E-08 $1.92 \mathrm{E}-06 \quad 8.66$

$1.04 \mathrm{E}-07 \quad 2.65 \mathrm{E}-06 \quad 8.66$
Table 10 Genes differentially expressed in skin T effector cells of SPF + SA-colonized mice vs. SPF-colonized mice (Continued)

\begin{tabular}{lllll}
\hline Gene & Log2FoldChange & $p$ value & $p$ adj & absFC \\
\hline Jmjd8 & 8.66 & $5.71 \mathrm{E}-10$ & $2.80 \mathrm{E}-08$ & 8.66
\end{tabular}

RP23-37809.1 8.65

7.83E-09 2.85E-07 8.65

Zfp212 8.63

$1.91 \mathrm{E}-07 \quad 4.45 \mathrm{E}-06 \quad 8.63$

Acvr1 8.63

Zdhhc15 $\quad 8.63$

4.06E-07 $8.51 \mathrm{E}-06 \quad 8.63$

$8.13 \mathrm{E}-10 \quad 3.68 \mathrm{E}-08 \quad 8.63$

6.23E-09 2.34E-07 8.62

$5.24 \mathrm{E}-06 \quad 7.60 \mathrm{E}-05 \quad 8.62$

7.00E-06 9.67E-05 8.62

1.33E-06 2.39E-05 8.62

$9.62 \mathrm{E}-12 \quad 7.15 \mathrm{E}-10 \quad 8.62$

3.62E-10 1.84E-08 8.62

$2.23 \mathrm{E}-17 \quad 6.86 \mathrm{E}-15 \quad 8.61$

$7.75 \mathrm{E}-07 \quad 1.48 \mathrm{E}-05 \quad 8.61$

$2.02 \mathrm{E}-13 \quad 2.45 \mathrm{E}-11 \quad 8.61$

3.93E-13 4.43E-11 8.61

$1.98 \mathrm{E}-06 \quad 3.31 \mathrm{E}-05 \quad 8.60$

$3.14 \mathrm{E}-07 \quad 6.84 \mathrm{E}-06 \quad 8.59$

3.55E-10 1.81E-08 8.58

$\begin{array}{lll}7.32 \mathrm{E}-09 & 2.70 \mathrm{E}-07 \quad 8.58\end{array}$

$1.09 \mathrm{E}-07 \quad 2.75 \mathrm{E}-06 \quad 8.58$

$7.78 \mathrm{E}-10 \quad 3.56 \mathrm{E}-08 \quad 8.58$

7.74E-10 3.56E-08 8.58

2.42E-09 9.93E-08 8.58

$9.01 \mathrm{E}-18 \quad 3.10 \mathrm{E}-15 \quad 8.57$

$1.86 \mathrm{E}-07 \quad 4.36 \mathrm{E}-06 \quad 8.56$

$2.48 \mathrm{E}-08 \quad 7.66 \mathrm{E}-07 \quad 8.56$

$2.98 \mathrm{E}-15 \quad 5.30 \mathrm{E}-13 \quad 8.56$

3.26E-06 5.04E-05 8.55

1.33E-07 3.24E-06 8.55

$1.46 \mathrm{E}-07 \quad 3.52 \mathrm{E}-06 \quad 8.55$

5.49E-09 2.09E-07 8.54

$2.10 \mathrm{E}-07 \quad 4.79 \mathrm{E}-06 \quad 8.54$

6.99E-06 9.67E-05 8.54

6.01E-08 1.65E-06 8.53

$2.95 \mathrm{E}-09 \quad 1.18 \mathrm{E}-07 \quad 8.53$

$6.18 \mathrm{E}-11 \quad 3.76 \mathrm{E}-09 \quad 8.53$

$1.32 \mathrm{E}-08 \quad 4.43 \mathrm{E}-07 \quad 8.53$

$8.50 \mathrm{E}-10 \quad 3.83 \mathrm{E}-08 \quad 8.52$

$1.62 \mathrm{E}-07 \quad 3.84 \mathrm{E}-06 \quad 8.50$

6.43E-11 3.90E-09 8.50

7.29E-09 2.69E-07 8.50

2.87E-09 1.15E-07 8.49

$1.10 \mathrm{E}-07 \quad 2.78 \mathrm{E}-06 \quad 8.49$ 
Table 10 Genes differentially expressed in skin T effector cells of SPF + SA-colonized mice vs. SPF-colonized mice (Continued)

\begin{tabular}{lllll}
\hline Gene & Log2FoldChange & $p$ value & $p$ adj & absFC \\
\hline Chchd10 & 8.49 & $6.25 \mathrm{E}-10$ & $2.95 \mathrm{E}-08$ & 8.49
\end{tabular}

Nr6a1

8.48

Ni6a1

RP24-303G10.1 8.48

Exoc312 8.48

Gm15609 $\quad 8.47$

Gm17477 $\quad 8.47$

Egfl8

8.47

Pcdhb17

8.47

Shb

8.47

117

Slc1a3

8.46

Ntrk3

8.46

Gypa

8.46

Tmem255b

8.45

Tgfa

8.45

6430590A07Rik 8.43

Ltbp2

8.43

Gm17491

8.43

4-Sep

8.43

Mboat2

8.43

Tle2

8.42

Gjb3

8.42

Rassf10

8.42

Dnm3os

8.42

Tenm4

8.42

D630008014Rik 8.41

Enpp3

8.40

Kcna2

8.40

Gm15844

8.40

Tor4a

8.40

Trp63

8.39

Myold

8.39

Ctif

8.39

Calm4

8.39

Serpinb1c

8.39

RP24-421E18.7

8.38

Dgkh

8.38

Bnc2

8.38

Pfkfb2

8.38

Pcdh9

8.37

Abca12

8.37
$2.21 \mathrm{E}-07 \quad 4.99 \mathrm{E}-06 \quad 8.48$

$1.10 \mathrm{E}-08 \quad 3.82 \mathrm{E}-07 \quad 8.48$

$3.53 \mathrm{E}-10 \quad 1.81 \mathrm{E}-08 \quad 8.48$

2.05E-08 6.56E-07 8.48

$1.41 \mathrm{E}-06 \quad 2.50 \mathrm{E}-05 \quad 8.48$

$5.80 \mathrm{E}-10 \quad 2.80 \mathrm{E}-08 \quad 8.47$

6.01E-11 3.69E-09 8.47

$1.56 \mathrm{E}-11 \quad 1.08 \mathrm{E}-09 \quad 8.47$

$1.01 \mathrm{E}-07 \quad 2.59 \mathrm{E}-06 \quad 8.47$

$2.08 \mathrm{E}-08 \quad 6.63 \mathrm{E}-07 \quad 8.47$

$8.04 \mathrm{E}-11 \quad 4.75 \mathrm{E}-09 \quad 8.46$

3.99E-06 6.04E-05 8.46

4.16E-07 $\quad 8.66 \mathrm{E}-06 \quad 8.46$

4.66E-06 6.85E-05 8.45

$2.10 \mathrm{E}-05 \quad 2.44 \mathrm{E}-04 \quad 8.45$

5.81E-10 2.80E-08 8.44

$9.61 \mathrm{E}-10 \quad 4.28 \mathrm{E}-08 \quad 8.43$

2.02E-07 4.65E-06 8.43

3.53E-07 7.55E-06 8.43

$1.00 \mathrm{E}-23 \quad 9.51 \mathrm{E}-21 \quad 8.43$

$1.44 \mathrm{E}-07 \quad 3.50 \mathrm{E}-06 \quad 8.43$

$1.11 \mathrm{E}-08 \quad 3.84 \mathrm{E}-07 \quad 8.42$

2.70E-05 3.01E-04 8.42

1.73E-08 5.62E-07 8.42

$2.43 \mathrm{E}-12 \quad 2.16 \mathrm{E}-10 \quad 8.42$

$6.98 \mathrm{E}-09 \quad 2.60 \mathrm{E}-07 \quad 8.42$

4.49E-06 6.65E-05 8.41

$6.18 \mathrm{E}-09 \quad 2.32 \mathrm{E}-07 \quad 8.40$

9.09E-07 1.71E-05 8.40

$2.84 \mathrm{E}-05 \quad 3.12 \mathrm{E}-04 \quad 8.40$

1.53E-06 2.67E-05 8.40

$1.37 \mathrm{E}-12 \quad 1.33 \mathrm{E}-10 \quad 8.39$

$1.02 \mathrm{E}-05 \quad 1.34 \mathrm{E}-04 \quad 8.39$

$1.18 \mathrm{E}-05 \quad 1.51 \mathrm{E}-04 \quad 8.39$

$8.28 \mathrm{E}-08 \quad 2.17 \mathrm{E}-06 \quad 8.39$

6.27E-12 5.09E-10 8.38

$8.78 \mathrm{E}-12 \quad 6.65 \mathrm{E}-10 \quad 8.38$

3.63E-11 2.34E-09 8.38

$1.28 \mathrm{E}-08 \quad 4.31 \mathrm{E}-07 \quad 8.38$

1.06E-07 2.69E-06 8.37

1.07E-06 1.97E-05 8.37
$6.62 \mathrm{E}-12 \quad 5.34 \mathrm{E}-10 \quad 8.39$
Table 10 Genes differentially expressed in skin T effector cells of SPF + SA-colonized mice vs. SPF-colonized mice (Continued)

\begin{tabular}{lllll}
\hline Gene & Log2FoldChange & $p$ value & $p$ adj & absFC \\
\hline Fzd4 & 8.37 & $8.16 \mathrm{E}-09$ & $2.96 \mathrm{E}-07$ & 8.37
\end{tabular}

Csrnp2

8.35

$1.68 \mathrm{E}-08 \quad 5.49 \mathrm{E}-07 \quad 8.35$

Cds1

8.35

Tnn

$2.64 \mathrm{E}-09 \quad 1.07 \mathrm{E}-07 \quad 8.35$

Kcna5

8.35

Fermt1

8.35

$1.54 \mathrm{E}-05 \quad 1.89 \mathrm{E}-04 \quad 8.35$

$1.78 \mathrm{E}-09 \quad 7.56 \mathrm{E}-08 \quad 8.35$

$9.76 \mathrm{E}-07 \quad 1.82 \mathrm{E}-05 \quad 8.34$

Comp

3.31E-08 9.89E-07 8.34

Pkp1

$1.93 \mathrm{E}-09 \quad 8.11 \mathrm{E}-08 \quad 8.34$

Hephl1

$6.29 \mathrm{E}-06 \quad 8.88 \mathrm{E}-05 \quad 8.33$

Nxpe4

2.67E-09 1.08E-07 8.33

Prkd1

$1.70 \mathrm{E}-06 \quad 2.91 \mathrm{E}-05 \quad 8.33$

Gm7162 $\quad 8.33$

Tfap2b

3.33E-09 1.32E-07 8.33

2.37E-07 5.28E-06 8.33

RP24-496C22.5 8.33

Ptprr 8.33

Cacna1c $\quad 8.33$

Fam57b $\quad 8.32$

RP24-360B3.2 8.32

Gtf2ird1 $\quad 8.31$

Tnfrsf22 8.31

P3h1 8.30

3110001122Rik $\quad 8.30$

Dmpk $\quad 8.30$

Tmem41a $\quad 8.30$

Hoxa5 $\quad 8.30$

Myocd $\quad 8.30$

Ackr1 8.30

Sox6

8.29

Schip1_dup1 8.29

Tc2n

8.29

Ptpn14 8.29

Zglp1

8.29

Trmt11

8.29

$\mathrm{C} 2 \mathrm{~cd} 2 \mathrm{l} \quad 8.28$

RP23-33315.3 $\quad 8.28$

Pkhd111 $\quad 8.28$

Nxpe2 $\quad 8.28$

Tex15 8.28

Syt7 $\quad 8.27$

Mmgt2 $\quad 8.26$

$\begin{array}{ll}\text { Al838599 } & 8.26\end{array}$

Prr9 8.26

Srd5a3

8.25
2.49E-09 1.01E-07 8.33

3.74E-05 3.95E-04 8.33

9.05E-10 4.04E-08 8.33

3.82E-09 1.50E-07 8.32

$1.88 \mathrm{E}-12 \quad 1.73 \mathrm{E}-10 \quad 8.32$

$2.43 \mathrm{E}-11 \quad 1.62 \mathrm{E}-09 \quad 8.31$

$2.04 \mathrm{E}-10 \quad 1.08 \mathrm{E}-08 \quad 8.31$

$1.23 \mathrm{E}-07 \quad 3.03 \mathrm{E}-06 \quad 8.30$

5.23E-07 1.06E-05 8.30

$5.10 \mathrm{E}-13 \quad 5.52 \mathrm{E}-11 \quad 8.30$

$1.01 \mathrm{E}-11 \quad 7.44 \mathrm{E}-10 \quad 8.30$

$2.15 \mathrm{E}-07 \quad 4.88 \mathrm{E}-06 \quad 8.30$

3.07E-08 9.24E-07 $\quad 8.30$

$1.04 \mathrm{E}-11 \quad 7.65 \mathrm{E}-10 \quad 8.30$

1.95E-06 3.27E-05 8.29

7.42E-08 1.99E-06 8.29

3.43E-09 1.36E-07 8.29

$5.58 \mathrm{E}-10 \quad 2.76 \mathrm{E}-08 \quad 8.29$

9.16E-08 2.37E-06 8.29

1.62E-09 6.91E-08 8.29

$2.57 \mathrm{E}-08 \quad 7.91 \mathrm{E}-07 \quad 8.28$

3.72E-06 5.67E-05 8.28

$1.13 \mathrm{E}-07 \quad 2.85 \mathrm{E}-06 \quad 8.28$

6.37E-07 1.25E-05 8.28

$6.48 \mathrm{E}-08 \quad 1.77 \mathrm{E}-06 \quad 8.28$

2.03E-08 6.49E-07 8.27

$1.85 \mathrm{E}-08 \quad 5.96 \mathrm{E}-07 \quad 8.26$

1.20E-07 2.97E-06 8.26

$2.11 \mathrm{E}-05 \quad 2.45 \mathrm{E}-04 \quad 8.26$

$1.54 \mathrm{E}-09 \quad 6.59 \mathrm{E}-08 \quad 8.25$ 
Table 10 Genes differentially expressed in skin T effector cells of SPF + SA-colonized mice vs. SPF-colonized mice (Continued)

\begin{tabular}{|c|c|c|c|c|}
\hline Gene & Log2FoldChange & $p$ value & $p$ adj & absFC \\
\hline Slc35f1 & 8.25 & $5.82 \mathrm{E}-09$ & $2.20 \mathrm{E}-07$ & 8.25 \\
\hline Tmtc4 & 8.25 & 1.67E-05 & $2.01 \mathrm{E}-04$ & 8.25 \\
\hline Сyp2e1 & 8.25 & $9.70 \mathrm{E}-07$ & $1.81 \mathrm{E}-05$ & 8.25 \\
\hline RP23-157G2.2 & 8.24 & $2.86 \mathrm{E}-08$ & 8.66E-07 & 8.24 \\
\hline Plin4 & 8.23 & 4.95E-08 & 1.40E-06 & 8.23 \\
\hline Heatr5b & 8.23 & $7.49 \mathrm{E}-09$ & $2.76 \mathrm{E}-07$ & 8.23 \\
\hline Lhx6 & 8.22 & $2.28 \mathrm{E}-05$ & $2.62 \mathrm{E}-04$ & 8.22 \\
\hline Ccdc85a & 8.22 & $6.36 \mathrm{E}-08$ & 1.74E-06 & 8.22 \\
\hline RP23-463H10.1 & 8.21 & $1.18 \mathrm{E}-06$ & $2.15 \mathrm{E}-05$ & 8.21 \\
\hline RP23-465A17.7 & 8.21 & $5.93 \mathrm{E}-08$ & $1.64 \mathrm{E}-06$ & 8.21 \\
\hline Pof1b & 8.21 & $5.35 \mathrm{E}-06$ & $7.75 \mathrm{E}-05$ & 8.21 \\
\hline Vwa3a & 8.21 & 1.65E-05 & $2.00 \mathrm{E}-04$ & 8.21 \\
\hline Rhbdd2 & 8.20 & $6.07 \mathrm{E}-10$ & $2.89 \mathrm{E}-08$ & 8.20 \\
\hline Zfp94 & 8.19 & $2.52 \mathrm{E}-09$ & $1.03 \mathrm{E}-07$ & 8.19 \\
\hline Zp1 & 8.18 & $6.28 \mathrm{E}-07$ & $1.23 \mathrm{E}-05$ & 8.18 \\
\hline Gm38142 & 8.18 & $1.79 \mathrm{E}-08$ & $5.80 \mathrm{E}-07$ & 8.18 \\
\hline Fam174b & 8.18 & $6.66 \mathrm{E}-12$ & 5.35E-10 & 8.18 \\
\hline Gm37399 & 8.18 & $7.40 \mathrm{E}-07$ & $1.42 \mathrm{E}-05$ & 8.18 \\
\hline Lrig3 & 8.17 & $1.02 \mathrm{E}-08$ & $3.60 \mathrm{E}-07$ & 8.17 \\
\hline Tcea2 & 8.17 & $1.89 \mathrm{E}-06$ & $3.18 \mathrm{E}-05$ & 8.17 \\
\hline Gm20696 & 8.15 & $1.01 \mathrm{E}-05$ & 1.32E-04 & 8.15 \\
\hline Sfxn4 & 8.15 & $5.76 \mathrm{E}-10$ & $2.80 \mathrm{E}-08$ & 8.15 \\
\hline Prom1 & 8.15 & $8.89 \mathrm{E}-06$ & 1.19E-04 & 8.15 \\
\hline Has2 & 8.15 & $2.20 \mathrm{E}-08$ & $6.92 \mathrm{E}-07$ & 8.15 \\
\hline Mamstr & 8.14 & $3.33 \mathrm{E}-08$ & $9.93 \mathrm{E}-07$ & 8.14 \\
\hline Cadps2 & 8.14 & $2.57 \mathrm{E}-07$ & $5.68 \mathrm{E}-06$ & 8.14 \\
\hline Fignl2 & 8.14 & $3.52 \mathrm{E}-09$ & 1.39E-07 & 8.14 \\
\hline II17rd & 8.14 & $1.77 \mathrm{E}-10$ & $9.49 \mathrm{E}-09$ & 8.14 \\
\hline Susd4 & 8.14 & 7.03E-07 & 1.36E-05 & 8.14 \\
\hline Spock1 & 8.13 & $3.04 \mathrm{E}-06$ & 4.75E-05 & 8.13 \\
\hline Ptger2 & 8.13 & $6.56 \mathrm{E}-07$ & $1.28 \mathrm{E}-05$ & 8.13 \\
\hline Nudt12 & 8.13 & $9.58 \mathrm{E}-07$ & $1.79 \mathrm{E}-05$ & 8.13 \\
\hline Frem2 & 8.13 & $1.84 \mathrm{E}-06$ & $3.11 \mathrm{E}-05$ & 8.13 \\
\hline Wfdc3 & 8.13 & $1.05 \mathrm{E}-05$ & 1.37E-04 & 8.13 \\
\hline Gpr4 & 8.12 & $3.49 \mathrm{E}-05$ & $3.72 \mathrm{E}-04$ & 8.12 \\
\hline Akap6 & 8.12 & $2.24 \mathrm{E}-06$ & $3.65 \mathrm{E}-05$ & 8.12 \\
\hline Gprasp2 & 8.11 & $1.27 \mathrm{E}-08$ & 4.30E-07 & 8.11 \\
\hline Sncaip & 8.11 & $2.29 \mathrm{E}-05$ & $2.63 \mathrm{E}-04$ & 8.11 \\
\hline Prkab1 & 8.11 & $7.89 \mathrm{E}-24$ & $8.32 \mathrm{E}-21$ & 8.11 \\
\hline Gm37780 & 8.11 & 1.17E-05 & 1.50E-04 & 8.11 \\
\hline Unc5c & 8.10 & $2.23 \mathrm{E}-07$ & $5.03 \mathrm{E}-06$ & 8.10 \\
\hline Gm37648 & 8.09 & $2.13 \mathrm{E}-06$ & $3.50 \mathrm{E}-05$ & 8.09 \\
\hline Gkn3 & 8.09 & 4.75E-05 & 4.83E-04 & 8.09 \\
\hline
\end{tabular}

Table 10 Genes differentially expressed in skin T effector cells of SPF + SA-colonized mice vs. SPF-colonized mice (Continued)

\begin{tabular}{|c|c|c|c|c|}
\hline Gene & Log2FoldChange & $p$ value & $p$ adj & $\mathrm{absFC}$ \\
\hline St6galnac2 & 8.09 & $4.03 \mathrm{E}-05$ & $4.21 \mathrm{E}-04$ & 8.09 \\
\hline Klhl23 & 8.08 & $2.90 \mathrm{E}-08$ & $8.78 \mathrm{E}-07$ & 8.08 \\
\hline Olfr78 & 8.07 & $3.25 \mathrm{E}-07$ & $7.05 \mathrm{E}-06$ & 8.07 \\
\hline RP24-247A21.1 & 8.07 & $2.02 \mathrm{E}-05$ & $2.36 \mathrm{E}-04$ & 8.07 \\
\hline Dos & 8.07 & $1.49 \mathrm{E}-11$ & $1.05 E-09$ & 8.07 \\
\hline Scn3a & 8.07 & $6.89 \mathrm{E}-11$ & 4.11E-09 & 8.07 \\
\hline Jade3 & 8.06 & $3.68 \mathrm{E}-06$ & $5.62 \mathrm{E}-05$ & 8.06 \\
\hline Fam110b & 8.06 & 4.07E-06 & $6.12 \mathrm{E}-05$ & 8.06 \\
\hline 4930578C19Rik & 8.06 & $4.64 \mathrm{E}-06$ & $6.82 \mathrm{E}-05$ & 8.06 \\
\hline Tmed8 & 8.06 & $1.87 \mathrm{E}-07$ & 4.37E-06 & 8.06 \\
\hline Hdhd3 & 8.04 & 1.83E-09 & $7.76 \mathrm{E}-08$ & 8.04 \\
\hline RP24-147H20.3 & 8.04 & $7.10 \mathrm{E}-05$ & $6.78 \mathrm{E}-04$ & 8.04 \\
\hline Adamts20 & 8.04 & $1.81 \mathrm{E}-05$ & $2.15 \mathrm{E}-04$ & 8.04 \\
\hline B3gnt3 & 8.04 & $4.03 \mathrm{E}-07$ & $8.49 \mathrm{E}-06$ & 8.04 \\
\hline Mal2 & 8.03 & 3.77E-05 & $3.98 \mathrm{E}-04$ & 8.03 \\
\hline Tmem41b & 8.03 & $1.44 \mathrm{E}-16$ & $3.69 E-14$ & 8.03 \\
\hline Efhd1 & 8.03 & $1.47 \mathrm{E}-06$ & $2.60 \mathrm{E}-05$ & 8.03 \\
\hline Glce & 8.02 & $2.49 \mathrm{E}-06$ & $3.99 \mathrm{E}-05$ & 8.02 \\
\hline Rragd & 8.02 & $7.09 \mathrm{E}-06$ & $9.80 \mathrm{E}-05$ & 8.02 \\
\hline Vipr2 & 8.02 & $6.53 \mathrm{E}-06$ & $9.15 E-05$ & 8.02 \\
\hline $\mathrm{Htr} 7$ & 8.02 & $8.62 \mathrm{E}-07$ & $1.63 \mathrm{E}-05$ & 8.02 \\
\hline Hbb-bt & 8.02 & $2.48 \mathrm{E}-15$ & $4.56 \mathrm{E}-13$ & 8.02 \\
\hline Wipf3 & 8.01 & $1.35 \mathrm{E}-06$ & $2.41 \mathrm{E}-05$ & 8.01 \\
\hline Gm14085 & 8.01 & $3.05 E-10$ & $1.58 \mathrm{E}-08$ & 8.01 \\
\hline Al846148 & 8.01 & $2.64 \mathrm{E}-06$ & $4.18 \mathrm{E}-05$ & 8.01 \\
\hline Unc13b & 7.99 & $1.05 \mathrm{E}-07$ & $2.68 \mathrm{E}-06$ & 7.99 \\
\hline Gm37519 & 7.99 & 6.67E-09 & $2.49 \mathrm{E}-07$ & 7.99 \\
\hline Dnah6 & 7.98 & $1.82 \mathrm{E}-06$ & $3.09 E-05$ & 7.98 \\
\hline Zdbf2 & 7.97 & $3.06 \mathrm{E}-06$ & $4.78 \mathrm{E}-05$ & 7.97 \\
\hline Chodl & 7.97 & 4.73E-05 & $4.81 \mathrm{E}-04$ & 7.97 \\
\hline Tbc1d19 & 7.97 & 4.07E-06 & $6.12 \mathrm{E}-05$ & 7.97 \\
\hline Aoc3 & 7.97 & $5.53 \mathrm{E}-07$ & $1.11 \mathrm{E}-05$ & 7.97 \\
\hline Lgr6 & 7.97 & $5.73 \mathrm{E}-06$ & $8.18 \mathrm{E}-05$ & 7.97 \\
\hline Prss36 & 7.96 & $3.98 \mathrm{E}-08$ & $1.17 \mathrm{E}-06$ & 7.96 \\
\hline Zcchc18 & 7.96 & 1.70E-05 & $2.04 \mathrm{E}-04$ & 7.96 \\
\hline Ak4 & 7.95 & $3.57 \mathrm{E}-05$ & $3.80 \mathrm{E}-04$ & 7.95 \\
\hline Pde4c & 7.95 & $1.04 \mathrm{E}-07$ & $2.66 \mathrm{E}-06$ & 7.95 \\
\hline Ring1 & 7.95 & $7.44 \mathrm{E}-10$ & $3.47 \mathrm{E}-08$ & 7.95 \\
\hline Kenb1 & 7.95 & 1.15E-07 & $2.88 \mathrm{E}-06$ & 7.95 \\
\hline Rergl & 7.94 & $7.72 \mathrm{E}-05$ & $7.24 \mathrm{E}-04$ & 7.94 \\
\hline Ccdc67 & 7.94 & $3.42 \mathrm{E}-07$ & $7.34 \mathrm{E}-06$ & 7.94 \\
\hline Ptx3 & 7.94 & $2.26 \mathrm{E}-06$ & $3.66 \mathrm{E}-05$ & 7.94 \\
\hline $\mathrm{Cc} 2 \mathrm{~d} 2 \mathrm{a}$ & 7.93 & 4.84E-10 & $2.42 \mathrm{E}-08$ & 7.93 \\
\hline
\end{tabular}


Table 10 Genes differentially expressed in skin T effector cells of SPF + SA-colonized mice vs. SPF-colonized mice (Continued)

\begin{tabular}{|c|c|c|c|c|}
\hline Gene & Log2FoldChange & $p$ value & $p$ adj & absFC \\
\hline Efcab7 & 7.93 & $9.20 \mathrm{E}-06$ & $1.23 \mathrm{E}-04$ & 7.93 \\
\hline Gm436 & 7.92 & 1.19E-04 & $1.03 \mathrm{E}-03$ & 7.92 \\
\hline Srd5a1 & 7.92 & $6.83 \mathrm{E}-07$ & $1.32 \mathrm{E}-05$ & 7.92 \\
\hline Stac & 7.92 & 3.77E-06 & $5.74 \mathrm{E}-05$ & 7.92 \\
\hline Pbld2 & 7.92 & $5.53 \mathrm{E}-07$ & $1.11 \mathrm{E}-05$ & 7.92 \\
\hline Atoh8 & 7.91 & $1.55 \mathrm{E}-06$ & $2.69 \mathrm{E}-05$ & 7.91 \\
\hline Dhrs13 & 7.91 & $1.26 \mathrm{E}-08$ & 4.27E-07 & 7.91 \\
\hline Hoxb7 & 7.91 & $1.20 \mathrm{E}-06$ & 2.17E-05 & 7.91 \\
\hline Cox18 & 7.90 & 1.50E-10 & $8.21 \mathrm{E}-09$ & 7.90 \\
\hline B430010l23Rik & 7.90 & $5.76 \mathrm{E}-10$ & $2.80 \mathrm{E}-08$ & 7.90 \\
\hline Acaa1b & 7.90 & $5.23 \mathrm{E}-07$ & $1.06 \mathrm{E}-05$ & 7.90 \\
\hline Micall2 & 7.90 & $3.92 \mathrm{E}-06$ & $5.94 \mathrm{E}-05$ & 7.90 \\
\hline Galns & 7.89 & $2.87 \mathrm{E}-06$ & $4.51 \mathrm{E}-05$ & 7.89 \\
\hline RP24-496C22.2 & 7.89 & $8.04 \mathrm{E}-06$ & $1.10 \mathrm{E}-04$ & 7.89 \\
\hline Armcx5 & 7.88 & $7.69 \mathrm{E}-11$ & 4.57E-09 & 7.88 \\
\hline Sox17 & 7.87 & $5.06 \mathrm{E}-07$ & $1.04 \mathrm{E}-05$ & 7.87 \\
\hline Tmem110 & 7.87 & $5.65 \mathrm{E}-14$ & $7.88 \mathrm{E}-12$ & 7.87 \\
\hline C130023A14Rik & 7.87 & 2.61E-11 & $1.71 \mathrm{E}-09$ & 7.87 \\
\hline RP23-293 K21.1 & 7.87 & 1.65E-06 & $2.84 \mathrm{E}-05$ & 7.87 \\
\hline $\mathrm{C} \times 3 \mathrm{Cl} 1$ & 7.86 & $6.88 \mathrm{E}-08$ & 1.87E-06 & 7.86 \\
\hline Atat1 & 7.86 & $5.25 E-05$ & $5.25 E-04$ & 7.86 \\
\hline Dsg2 & 7.86 & $3.85 \mathrm{E}-07$ & $8.13 \mathrm{E}-06$ & 7.86 \\
\hline Aldh1a7 & 7.86 & 1.04E-04 & $9.22 \mathrm{E}-04$ & 7.86 \\
\hline Zfp69 & 7.85 & 2.09E-06 & $3.45 \mathrm{E}-05$ & 7.85 \\
\hline Myh14 & 7.85 & $6.81 \mathrm{E}-07$ & $1.32 \mathrm{E}-05$ & 7.85 \\
\hline Afap1l1 & 7.85 & $3.18 \mathrm{E}-26$ & $5.97 \mathrm{E}-23$ & 7.85 \\
\hline Mpzl2 & 7.85 & $6.86 \mathrm{E}-06$ & $9.54 \mathrm{E}-05$ & 7.85 \\
\hline Flywch2 & 7.84 & $2.73 \mathrm{E}-08$ & $8.32 \mathrm{E}-07$ & 7.84 \\
\hline Krtcap3 & 7.84 & $2.53 \mathrm{E}-05$ & $2.86 \mathrm{E}-04$ & 7.84 \\
\hline Epb4.114b & 7.83 & 1.30E-05 & $1.64 \mathrm{E}-04$ & 7.83 \\
\hline Ficd & 7.83 & $3.45 \mathrm{E}-06$ & 5.31E-05 & 7.83 \\
\hline Sh3gl3 & 7.83 & 4.95E-06 & $7.21 \mathrm{E}-05$ & 7.83 \\
\hline Cyb561 & 7.83 & $2.01 \mathrm{E}-06$ & $3.35 \mathrm{E}-05$ & 7.83 \\
\hline Gm7909 & 7.83 & $1.84 \mathrm{E}-07$ & 4.31E-06 & 7.83 \\
\hline Erf & 7.83 & 2.74E-08 & $8.34 \mathrm{E}-07$ & 7.83 \\
\hline Scgb3a1 & 7.82 & $8.59 E-07$ & $1.62 \mathrm{E}-05$ & 7.82 \\
\hline Cwh43 & 7.82 & 4.37E-08 & $1.25 \mathrm{E}-06$ & 7.82 \\
\hline Tfap2e & 7.82 & $5.73 E-08$ & $1.59 \mathrm{E}-06$ & 7.82 \\
\hline Pou6f1 & 7.82 & 4.56E-06 & $6.72 \mathrm{E}-05$ & 7.82 \\
\hline Plxna2 & 7.82 & $6.16 \mathrm{E}-20$ & $3.25 E-17$ & 7.82 \\
\hline Fstl4 & 7.82 & 4.96E-08 & $1.40 \mathrm{E}-06$ & 7.82 \\
\hline Lmln & 7.81 & 4.34E-08 & $1.25 E-06$ & 7.81 \\
\hline RP24-560A18.1 & 7.81 & $3.62 \mathrm{E}-06$ & 5.55E-05 & 7.81 \\
\hline
\end{tabular}

Table 10 Genes differentially expressed in skin T effector cells of SPF + SA-colonized mice vs. SPF-colonized mice (Continued)

\begin{tabular}{lllll}
\hline Gene & Log2FoldChange & $p$ value & $p$ adj & absFC \\
\hline Prkg1 & 7.81 & $4.28 \mathrm{E}-06$ & $6.38 \mathrm{E}-05$ & 7.81 \\
RP24-399A15.2 & 7.80 & $2.45 \mathrm{E}-05$ & $2.79 \mathrm{E}-04$ & 7.80 \\
Cspg4 & 7.80 & $1.05 \mathrm{E}-12$ & $1.06 \mathrm{E}-10$ & 7.80 \\
Tmem86a & 7.80 & $1.88 \mathrm{E}-05$ & $2.22 \mathrm{E}-04$ & 7.80 \\
TIl1 & 7.79 & $5.08 \mathrm{E}-05$ & $5.11 \mathrm{E}-04$ & 7.79 \\
Laptm4b & 7.79 & $7.70 \mathrm{E}-09$ & $2.82 \mathrm{E}-07$ & 7.79 \\
6430573F11Rik & 7.79 & $5.68 \mathrm{E}-07$ & $1.13 \mathrm{E}-05$ & 7.79 \\
Gm26603 & 7.79 & $1.02 \mathrm{E}-07$ & $2.61 \mathrm{E}-06$ & 7.79 \\
Ptk7 & 7.78 & $8.48 \mathrm{E}-08$ & $2.22 \mathrm{E}-06$ & 7.78 \\
Igsf9 & 7.78 & $8.54 \mathrm{E}-06$ & $1.15 \mathrm{E}-04$ & 7.78 \\
RP23-372C7.4 & 7.78 & $2.73 \mathrm{E}-05$ & $3.04 \mathrm{E}-04$ & 7.78 \\
Nol41 & 7.78 & $4.88 \mathrm{E}-08$ & $1.39 \mathrm{E}-06$ & 7.78 \\
Slc7a2 & 7.78 & $1.20 \mathrm{E}-11$ & $8.69 \mathrm{E}-10$ & 7.78 \\
Zfp52 & 7.77 & $2.22 \mathrm{E}-05$ & $2.56 \mathrm{E}-04$ & 7.77 \\
A730049H05Rik & 7.77 & $2.35 \mathrm{E}-05$ & $2.69 \mathrm{E}-04$ & 7.77 \\
Yars2 & 7.77 & $8.91 \mathrm{E}-08$ & $2.32 \mathrm{E}-06$ & 7.77 \\
Mc5r & 7.77 & $6.54 \mathrm{E}-05$ & $6.31 \mathrm{E}-04$ & 7.77 \\
Gm20699 & 7.76 & $1.54 \mathrm{E}-08$ & $5.07 \mathrm{E}-07$ & 7.76 \\
4933407K13Rik & 7.76 & $1.15 \mathrm{E}-04$ & $1.00 \mathrm{E}-03$ & 7.76 \\
Tbc1d8 & 7.76 & $2.86 \mathrm{E}-18$ & $1.03 \mathrm{E}-15$ & 7.76 \\
Gm9917 & 7.75 & $7.64 \mathrm{E}-09$ & $2.80 \mathrm{E}-07$ & 7.75 \\
Aplp1 & 7.75 & $5.73 \mathrm{E}-06$ & $8.18 \mathrm{E}-05$ & 7.75 \\
4933416E03Rik & -7.75 & $7.80 \mathrm{E}-04$ & $4.99 \mathrm{E}-03$ & 7.75 \\
Nfatc4 & 7.75 & $2.59 \mathrm{E}-11$ & $1.70 \mathrm{E}-09$ & 7.75 \\
Cpeb1 & 7.74 & $2.24 \mathrm{E}-08$ & $6.99 \mathrm{E}-07$ & 7.74 \\
Bahcc1 & 7.74 & $8.17 \mathrm{E}-07$ & $1.55 \mathrm{E}-05$ & 7.74 \\
Scarb1 & 7.74 & $3.98 \mathrm{E}-17$ & $1.14 \mathrm{E}-14$ & 7.74 \\
\hline & & & & \\
\hline
\end{tabular}

activation of Th17 response, which might contribute to IL-17-driven inflammation in psoriasis.

\section{Discussion}

In this study, we profiled the skin microbiota of psoriasis patients and healthy controls using the $\mathrm{NIH}$ standardized protocol and with higher sequencing depth to gain a more comprehensive understanding in psoriasis-associated microbiome. Our data demonstrate that the psoriasis skin microbiome is more heterogeneous compared to that of healthy skin. The compositional variance in the psoriatic skin community could be attributable to local environmental changes that accompany or immediately precede psoriatic disease. Proliferating keratinocytes in psoriasis patients are a rich source of antimicrobial peptides such as LL37, $\beta$-defensin, and psoriasin [42]. The constant presence of these antimicrobial peptides could undermine equilibrium of the skin microbiome 

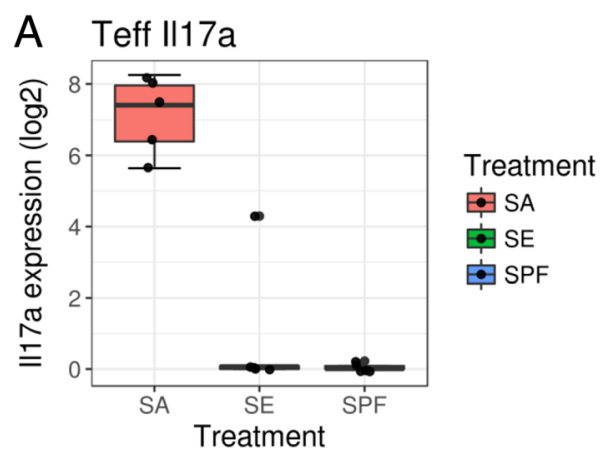

B Teff $\| 17 f$

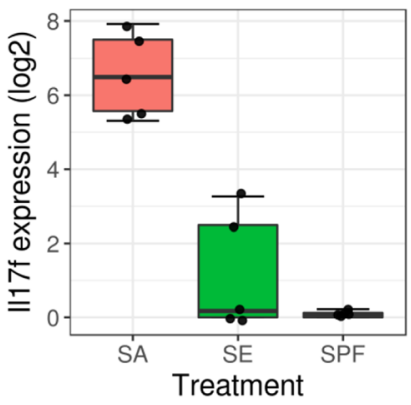

Treatment

由S

为 SE

由 SPF
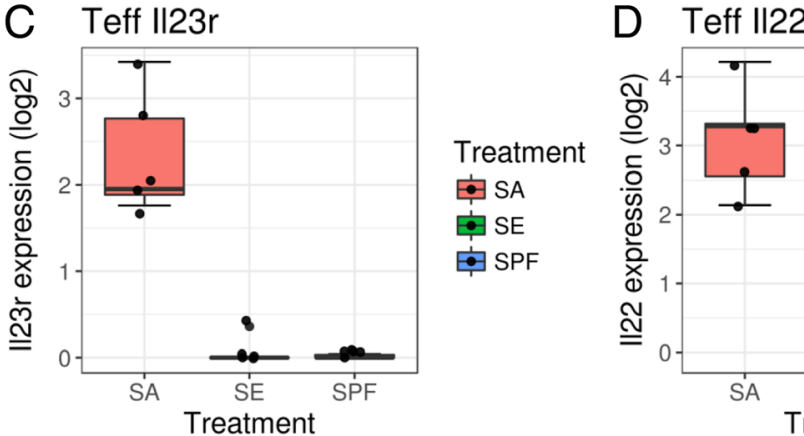

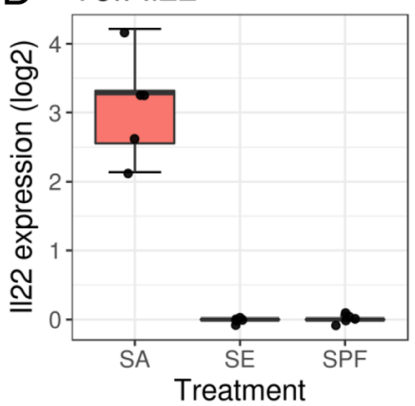

Treatment

宜SA

由S

目 SPF

Fig. 5 Staphylococcus aureus exposure triggers Th17 response in effector T cells. mRNA expression (log2FPKM) of cutaneous effector T cells from specific pathogen-free (SPF) mice colonized with Staphylococcus aureus (SA), Staphylococcus epidermidis (SE), or none (SPF). Compared to the SPF control, the Stapylococcus aureus colonization triggers gene expression in a IL-17A (adj $p$ value $=3.51 \mathrm{e}-7$ ), $\mathbf{b} \mathrm{IL}-17 \mathrm{~F}$ (adj $p$ value $=3.08 \mathrm{e}-6$ ), $\mathbf{c}$ IL-23R (adj $p$ value $=3.74 \mathrm{e}-8$ ), and $\mathbf{d}$ IL-22 (adj $p$ value $=1.01 \mathrm{e} 09$ ). Colonization with Staphylococcus epidermidis does not trigger Th17 response

community and select for microbial species resistant to these antimicrobials. Based on our data, we speculate that a healthy skin microbial community consists of key stabilizer species, which may prevent growth of other species in the local microenvironment. In psoriatic skin, these stabilizer species may be outcompeted by invading pathogenic species and/or inhibited by chronic exposure to antimicrobial peptides, enabling colonization by pathogenic bacteria normally excluded from this niche. This could explain the higher heterogeneity that we observed in psoriatic skin. In contrast to our result, Alekseyenko et al. and Gao et al. observed decreased bacterial diversity in psoriatic skin compared with healthy skin $[12,14]$ while Fahlen et al. found no difference [13]. Consistent with all the previous studies, we observed a decrease in relative abundance of Actinobacter associated with psoriasis skin [12-14]. Similar to Fahlen et al., we observed overrepresentation of Proteobacteria in psoriasis skin while both Gao et al. and Alekseyenko et al. showed a reduced abundance of Proteobacteria in psoriasis skin. These discrepancies might be due to the inherent heterogeneity in microbiota composition observed on the skin of psoriatic patients or to different experimental designs. This highlights the need to use standardized protocols among different studies to enhance reproducibility and to allow for meta-analysis of study cohorts. It is important to note that all studies mentioned above including ours profile the skin microbial community using an OTU (operational taxonomic unit) approach which groups reads from part of the $16 \mathrm{~S}$ rRNA gene in order to account for artifact variance introduced by sequencing error. The major limitation of this approach is that by grouping different sequence variants, subtle inter-species variance can be sacrificed, which can reduce the resolution of taxonomical assignment at the species level [43]. Despite the inherent limitation of OTU-based profiling, we were still able to gain species insights from our dataset.

Increased colonization of $S$. aureus in psoriatic skin has been reported previously in several small studies, but only a few of these examined unaffected skin from psoriasis patients [44]. Our data revealed a significant increase of colonization in both psoriatic lesional and non-lesional sites compared to the baseline levels of $S$. aureus colonization found in healthy skin. This suggests that the increase in S. aureus is less likely a consequence of structural change in the skin from psoriasis but rather might be an important factor in initiating disease. Indeed, the potential role of bacterial infection in initiating and exacerbating psoriasis has been shown in Streptococcus infection $[9,10]$. Our data from a murine model of Staphylococcal skin colonization suggests that cutaneous exposure to $S$. aureus triggers a strong Th17-type response in skin Teff cells as evident by induction of IL-17A, IL-17F, and IL22 cytokines. IL22 not only triggers a pro-inflammatory response, it also inhibits 
terminal differentiation of keratinocytes which is one of the characteristics of psoriasis. This suggests a potential capability of $S$. aureus to initiate psoriasis through upregulating a Th17 response. It is important to note that $S$. aureus consists of many strains and some strains are more virulent than others depending on their expression on a variety of different toxins and other molecules. Our murine experiments utilized S. aureus strain SF8300 from the USA300 lineage, which contains Panton-Valentine leukocidin (PVL) and phenol-soluble modulins (PSMs) contributing to its virulence in skin and soft tissue infections. Colonization of $S$. aureus has long been implicated in the pathogenesis of atopic dermatitis $[8,45]$. Consistent with a recent study demonstrating that the capacity to induce Th2-type inflammation is limited to specific $S$. aureus strains isolated from severe atopic dermatitis patients, we observed little induction in components of Th2 response except for GATA3, which is the transcription factor that is required for Th2 polarization [40]. Compared to the baseline level of $S$. aureus colonization in healthy controls, we found that $S$. aureus levels in some of the psoriasis samples were increased up to 90 -fold. The increased $S$. aureus colonization in psoriasis could be due to the expansion of S. aureus strains seen in healthy controls or colonization with new $S$. aureus strains. However, due to lack of strain resolution of $16 \mathrm{~S}$ rRNA-based profiling, we are not able to distinguish these two possibilities. In addition to strainspecific immunomodulating effects, our observation might suggest a temporal relationship between Th17 and Th2 polarization in response to $S$. aureus colonization and/or during neonatal development, as has been reported in murine models of atopic dermatitis [46]. Future studies examining the $S$. aureus strain diversity in psoriasis skin and skin immune response to $S$. aureus strains specifically isolated from patients with psoriasis would be of significant interest in further dissecting the role of this bacterium in psoriasis disease pathogenesis.

Propionibacterium is one of the most dominant skin commensal bacteria [24]. P. acnes has long been linked to acne vulgaris $[47,48]$, but recent studies suggest that $P$. acnes is also highly abundant in healthy skin and specific pathogenic $P$. acnes strains is one of the key determinants for acne vulgaris [49-51]. In this study, we found Propionibacterium to be abundant in healthy, lesional, and non-lesional skin but with highest abundance in healthy skin. Moreover, $P$. acnes and P. granulosum were two of the strongest microbial species associated with healthy skin. A possible explanation for the reduced abundance in Propionibacterium species in psoriatic lesions might be that reduced sebaceous content in psoriatic plaques contributes to a less favorable environment for Propionibacterium growth. The potential consequence of this reduction in Propionibacterium species in psoriasis is less clear. On one hand, $P$. acnes is known to produce propionate, a short chain fatty acid which can promote regulatory $\mathrm{T}$ cell in the colon [52], as well as RoxP (radical oxygenase of Propionibacterium acnes), which can potentially reduce oxidative stress and prevent skin inflammation [53]. In contrast, certain strains of $P$. acnes isolated from acne patients have the potential to induce higher IL-17 production compared to strains isolated from healthy subjects based on unknown mechanisms [54]. Although our current study does not have the resolution for $P$. acnes strain identification, identifying psoriasis-specific $P$. acnes isolates and compare them to those in healthy subject and assessing their differential genomic content and ability to modulate host $\mathrm{T}$ cell responses will be crucial in understanding whether the abundance or type of $P$. acnes in psoriasis patients contributes to their propensity for disease.

The association between Staphylococcus sciuri and psoriasis non-lesional skin is rather surprising since $S$. sciuri is better known as an animal-associated bacteria $[55,56]$. S. sciuri has also been found in the human skin in both healthy and hospitalized individuals [57]. The clinical relevance of $S$. sciuri has become important since several studies have isolated $S$. sciuri from hospitalized patients and methicillin-resistant strains of $S$. sciuri can be a health hazard for hospitalized patients [58-62]. Our study provides the first observation of $S$. sciuri in the context of psoriasis. It is possible that the S. sciuri carriers of our cohort obtained the bacteria from a previous hospital visit since $S$. sciuri has been found to be persistently present in the hospital environment [63]. While the possible role of $S$. sciuri in psoriasis is unclear, we have observed an interesting pattern of S. sciuri in our cohort. Our data show that an increase in S. sciuri abundance is exclusively associated with psoriasis skin, particularly in non-lesional skin (Fig. 3c). Moreover, $S$. sciuri abundance is negatively correlated with $P$. acnes, which is highly enriched in healthy skin (Fig. 4b). Together, our results suggest $S$. sciuri may have a potential role in psoriasis pathogenesis.

In order to understand the roles of microbiome in human health, it is important to consider the microbial community as whole. Bacterial interactions are as important as the host environment in shaping the skin microbial community. The microbe-microbe relationship can be competitive or symbiotic. We performed a correlation analysis on the most abundant microbial genera and species to elucidate possible microbemicrobe interactions in healthy and psoriasis skin. We found an anti-correlation between S. sciuri and $P$. acnes, consistent to their respective disease state associations. To our surprise, although $S$. aureus is enriched in psoriatic skin and is known from other studies to have a competitive relationship with $S$. epidermidis and $P$. acnes, our data did not corroborate these negative 
associations. Possible explanations for this might include strain-specific interactions as well as the impact of the skin environment on inter-species interactions, the latter being supported by our finding that inter-bacterial correlation clusters differed by different disease state. It is important to note that microbemicrobe relationships suggested in our study are only correlative, further experimentation on isolated microbes will be needed to validate these relationships. Nonetheless, our work predicts strong candidates for the microbe-microbe relationships that may be crucial for psoriasis pathogenesis. Taken together, our correlation analysis demonstrates the highly complex relationship among skin bacteria by showing that these inter-microbial relationships are altered in psoriasis, possibly due to changes in the biochemical changes in skin environment and/or ecological pressure imposed by an altered host immune response.

\section{Conclusion}

In this study, we adhered to a stringent sampling protocol and measured skin microbiome profiles associated with psoriasis skin at six different skin sites. Our data revealed higher diversity and heterogeneity in psoriatic skin relative to healthy skin. Taxonomic analyses revealed specific microbial signatures associated with each disease state at the genus and species levels. Intriguingly, we found Staphylococcus aureus to be more abundant in both psoriatic non-lesional and lesional skin while Staphylococcus epidermidis, Propionibacterium acnes, and Propionibacterium granulosum were more abundant in healthy skin. We further tested the impact of Staphylococcus aureus colonization on host response in murine skin and validated its capacity for Th17 polarization. Finally, we demonstrated that disease state can alter microbe-microbe interactions and co-associations possibly due to differences in the physical and chemical environment of the skin. Our study confirms that psoriasis is accompanied by a shift in the skin-resident microbial community and raises intriguing possibilities worthy of further exploration for how this might directly impact the host immune response and psoriasis pathogenesis.

\section{Methods}

\section{Study cohort}

Twenty-eight adult psoriasis patients and 26 healthy volunteers recruited from the San Francisco Bay area were enrolled in the study after providing informed consent. Individuals with abnormal coagulation studies, positive HIV screening test, or a known history of bleeding disorders, abdominal surgery, gastrointestinal cancer, inflammatory bowel disease, AIDS, or other immunosuppressive condition, or concurrent inflammatory skin condition were also excluded. All psoriasis patients had a diagnosis of psoriasis from a physician for at least 6 months prior to study enrollment, which was verified by study staff. To assess the psoriatic microbiome in an untreated state, subjects were excluded if they had received systemic biologic therapy in the last 6 months, non-biologic systemic medications (methotrexate, cyclosporine, corticosteroids, cyclophosphamide, retinoids, photochemotherapy) or antibiotics in the last month, or phototherapy or topical therapy in the last 2 weeks prior to skin swabbing. Healthy volunteers had no personal or family history of psoriasis.

\section{Specimen collection}

Skin swabs and stool samples were collected according to the protocol outlined in the Manual of Procedures for the NIH Human Microbiome Project [19]. Study participants were asked to refrain from showering and using any substances on their skin (lotion, perfume, make-up, etc.) for at least $24 \mathrm{~h}$ prior to skin swabbing. Samples of the skin microbiome were collected using individually packed, sterile cotton swabs (Epicentre Catch-All Swabs). For each subject, the skin was swabbed at six standardized sites: scalp, trunk, axilla, arm, leg, and gluteal fold for healthy samples and psoriasis unaffected samples. Psoriatic lesional samples were only taken when psoriasis plaques were present at one of the six sites. Negative controls were obtained by exposing swabs to room air for $10 \mathrm{~s}$. All samples were stored in $-80{ }^{\circ} \mathrm{C}$ while they awaited further processing.

\section{DNA sequencing}

DNA was extracted from the skin swab samples using the MasterPure Yeast DNA Purification Kit (Epicentre) with bead beating method to lyse the bacterial cells. To prepare skin microbiome library for sequencing, $16 \mathrm{~S}$ rRNA were amplified at V1 to V3 hypervariable region using a universal forward primer (V1_27F primer): 5'-AGAGTTTGATCCTGGCTCAG-3' attached to 5' Illumina adapter and indexed reverse primer (V3_534R primer): 5 '-ATTACCGCGGCTGCTGG-3' attached with 3' Illumina adapter sequence. Amplicon PCR reactions were completed as follows (per reaction): $2 \mu \mathrm{l}$ of gDNA, $1 \times$ final concentration of $10 \times$ LA PCR Buffer $\mathrm{ll}(\mathrm{Mg} 2+$ free) (Takara Bio USA), 0.4 mM dNTPs, 0.4 uM forward and reverse primers, $1.25 \mathrm{U}$ of TaKaRa LA Taq polymerase high fidelity (Takara Bio USA), and nuclease-free water to bring the final volume to $25 \mu$ l. PCR cycling protocol consisted of an initial denaturation of $5 \mathrm{~min}$ at $95^{\circ} \mathrm{C}, 30$ cycles of $30 \mathrm{~s}$ at $95{ }^{\circ} \mathrm{C}$, and $30 \mathrm{~s}$ at $56^{\circ} \mathrm{C}$ followed by $5 \mathrm{~min}$ at $72{ }^{\circ} \mathrm{C}$. PCR reactions were subsequently cleaned up using Agencourt AMPure XP kit (Beckman Coulter), and the purified amplicons were quantified using Quant-iT PicoGreen dsDNA Assay Kit (Invitrogen). Samples with amplicon concentrations less than three times above the average air control amplicon concentrations were 
excluded from the subsequent sequencing. In average, 60 70 samples were pooled in equal molar quantities and the pooled library was purified using minElute PCR purification kit (Qiagen) for the final purification. The pooled library was sequenced on a Miseq sequencer (Illumina) as described in commercially provided protocol with $25 \%$ phiX DNA added as spike-in. Miseq reagent kit V3 (Illumina) was used to generate paired-end 300-bp reads. To avoid confounding from batch effects or possible external contamination that could affect our comparison between psoriasis and healthy skin microbiome, each Miseq sequencing run was balanced with samples from three or four healthy subjects and four psoriasis samples.

\section{Data process and OTU picking}

After quality check, the high-quality pair-end reads were assembled into 550-bp fragments using FLASH [64] and we performed the subsequent analysis using Qiime scripts [65]. The pair-end-assembled sequence fragments were first aligned against the Qiime supplied reference database for picking close OTUs. Subsequently, the unaligned sequences clustered into $97 \%$ identity operational taxonomical units (OTUs) using UCLUST [66]. A representative sequence from each OTU cluster was aligned against the GreenGenes core set alignment template using PyNAST [67]. The chimera due to the PCR errors were identified by ChimeraSlayer and excluded from the subsequent analyses [68]. The remaining chimera-free OTUs were then used to approximate the phylogenetic tree using FastTree [69]. We removed samples with less than 10,000 sequences to ensure adequate sample depth after rarefaction. The chimera-free sequences were rarefied into 11,766 per samples using the custom script analyses (https://github.com/alifar76/ MicroNorm) to account for library size differences between samples.

\section{Subsequent analysis for community diversities, microbial signatures, and inter-microbial correlations}

The rarefied OTU table was used for all the subsequent analyses using Qiime 1.8.0 [65] and R [70]. Four alpha diversity metrics-chao1 index, observed OTUs, Shannon's diversity index, and Simpson's diversity index-were calculated for samples in each disease state, and the significance between different disease states were evaluated by a linear mixed effect model to account for multiple sampling from the same patient. The linear mixed effect model was performed by using $\mathrm{R}$ package lmerTest(v.3.0.1) [71]. The R package Kendall (v.2.2) [72] was used to perform the Mann-Kendall trend test to detect significant trends of alpha diversity across different disease states.

Weighted UniFrac dissimilarity matrices were calculated to determine beta diversity using Qiime script. To account for multiple sampling of different body sites from the same subject, we applied linear mixed effects model to the first principal component coordinate from weighted UniFrac (scripts available in Additional file 2). The heterogeneity of microbial communities within each disease state were determined by the average weighted UniFrac distances between site-matched samples within each disease state, and the significance was determined by one-way analysis of variance (ANOVA) followed by Tukey's multiple comparisons test done by GraphPad Prism 7 (statistics output in Additional file 3). The relative abundance of each microbial community was summarized by Qiime script at the phylum and genus level. The microbial signatures associated to each disease state at both genus and species level were identified by Lefse, which combines non-parametric Kruskal-Wallis test and linear discriminant analysis (LDA) [33]. Taxonomical features with $p$ value $<0.05$ and LDA effect size $>2.0$ were considered significant microbial signatures. To evaluate the skin site- and skin type-specific diversity changes and microbial signatures, the OTU tables were further subsetted into different skin sites or different skin types. The diversity and marker analyses were performed as described above. We further investigated the difference between psoriasis samples with high S. aureus abundance and those with low $S$. aureus abundance seen in healthy skin. We defined the highest $S$. aureus abundance in healthy skin as baseline. Psoriasis samples (both lesional and non-lesional) with higher S. aureus abundance than the baseline level were considered "S. aureus high" and psoriasis samples with lower or equal S. aureus abundance as the baseline level were considered " $S$. aureus low". Lefse analysis was performed to identify bacterial signatures associated with $S$. aureus high and $S$. aureus low samples. Lastly, the rarefied OTU table was summarized to genus (L6) and species (L7) by Qiime script and Spearman correlation was calculated using a $\mathrm{R}$ package, Hmisc (v. 4.1.1) [73], for the top 25 most abundant genera and top 30 most abundant species (scripts available in Additional file 4). The species-species correlations were further investigated within different disease states (scripts available in Additional file 4). Summarized taxonomic tables used in correlation analyses were available in Additional files 5 and 6.

\section{Mouse skin bacterial colonization}

C57BL/6 mice were purchased from Jackson Laboratories (Bar Harbor, ME) then bred and maintained in the UCSF-specific pathogen-free facility. All animal experiments were performed in accordance with the NIH Guide for the Care and Use of Laboratory Animals and the guidelines of the Laboratory Animal Resource Center and Institutional Animal Care and Use Committee of the University of California, San Francisco. 
Bacterial strains Staphylococcus epidermidis Tü3298 and Staphylococcus aureus SF8300 were grown in tryptic soy broth for 24-48 h; pelleted and cellular mass from $2.5 \mathrm{ml}$ of saturated culture was re-suspended in $100 \mu \mathrm{l}$ of PBS for colonization of each animal. Newborn C57BL6 mice were colonized starting on day 3 of life and every other day thereafter until post-natal day 19 by pipetting $100 \mu \mathrm{l}$ of bacterial suspension onto their skin and distributing evenly using a sterile PBS-soaked cotton-tipped swab.

\section{Isolation and RNA sequencing of CD4+ effector T (Teff) and regulatory $T$ (Treg) cells from skin of bacterially associated mice}

To isolate skin $\mathrm{T}$ cells for staining and FACS sorting, mice were sacrificed at 21 days of age and the entire trunk skin harvested and lightly defatted. The skin was then minced with scissors and re-suspended in a $50 \mathrm{ml}$ conical with 1-2 $\mathrm{ml}$ of digestion media comprised of $2 \mathrm{mg} / \mathrm{ml}$ collagenase XI, $0.5 \mathrm{mg} / \mathrm{ml}$ hyaluronidase, and $0.1 \mathrm{mg} / \mathrm{ml}$ DNase in RPMI with 1\% HEPES, 1\% penicillin-streptomycin, and $10 \%$ fetal calf serum. This mixture was incubated in a shaking incubated at $37{ }^{\circ} \mathrm{C}$ at $250 \mathrm{rpm}$ for $45 \mathrm{~min}$. An additional $15 \mathrm{ml}$ of RPMI/ HEPES/P-S/FCS media was then added, and the $50 \mathrm{ml}$ conical was shaken vigorously by hand for $30 \mathrm{~s}$. Another $15 \mathrm{ml}$ of media was added, and then, the entire suspension was filtered through a sterile $100-\mathrm{mm}$ cell strainer followed by a $40-\mathrm{mm}$ cell strainer into a new $50 \mathrm{ml}$ conical. The suspension was then pelleted, and the cell pellet was re-suspended in sort buffer (RPMI, 2 mM EDTA, $25 \mathrm{mM}$ HEPES, 2\% FBS) with U/ml RiboLock RNase inhibitor (Thermo Scientific) for staining for $30 \mathrm{~min}$ at $4^{\circ}$ $C$ with fluorophore-conjugated antibodies specific for CD3, CD4, CD8, CD25, CD45, ICOS, TCR $\beta$, and Tonbo Live-dead Ghost Dye. Teff (Live, CD45+, CD3int, CD4+, CD8neg, TCR $\beta+$ CD25neg, ICOSneg) cells were then isolated via cell sorting on a MoFlo XDP (Beckman Coulter) in the UCSF Flow Cytometry Core. Cells were pelleted and flash frozen. RNA isolation was performed by Expression Analysis Q2 Solutions using QIAGEN RNeasy Spin columns and was quantified via Nanodrop ND-8000 spectrophotometer. RNA quality was checked by Agilent Bioanalyzer Pico Chip. The SMARTer Ultra Low input kit was used to generate cDNA libraries which were then sequenced to a $25-\mathrm{M}$ read depth with Illumina RNASeq.

Reads were aligned to UCSC GRCm38/mm10 reference genome with STAR software (2.4.2a) [70]. SAM files were generated with SAMtools from alignment results [71]. Read counts were obtained with htseq-count (0.6.1p1) with the union option [72]. Differential expression was determined using the $\mathrm{R} /$ Bioconducter package
DESeq2 [73]. RPKM table was generated by Cuffdiff Cuffdiff (2.2.1) [74] (Additional file 7).

\section{Additional files}

\begin{abstract}
Additional file 1: Figure S1. Box plot shows the average weighted UniFrac distances among samples within each disease state at (a) arm, (b)trunk, (c)leg, (d)axilla, (e)gluteal fold and (f)scalp ( ${ }^{*}$ : $p$-value $<0.05$, **. $p$-value $<0.01^{* * *}$ : $p$-value $<0.001^{* * *}: p$-value $\left.<0.0001\right)$. Figure S2. Box plot shows the average weighted UniFrac distances among samples within each disease state in (a) dry and (b)moist skin group (*: $p$-value $<0.05, * *$ : $p$-value $<0.01^{* * *}$ : $p$-value $<0.001^{* * * *}$ : $p$-value $<0.0001$ ).

Figure S3. Relative abundance of $P$. acnes and $S$. aureus in each disease state at different body sites. Relative abundance of $\mathrm{P}$. acnes in healthy (red), psoriasis lesional (green), psoriasis non-lesional (blue) skin at (a) different skin sites and (b) different skin types (*: $p$-val $<0.05$, **: $\mathrm{p}$-val <0.01, ${ }^{* * * *}: \mathrm{p}$-val <0.0001). (c) Box plot showing S. aureus abundance in $\mathrm{S}$. aureus high samples. $\mathrm{S}$. aureus high samples were defined as samples with higher $\mathrm{S}$. aureus abundance than the highest $\mathrm{S}$. aureus abundance among the healthy samples (baseline $=0.0068$ ). $(\mathrm{d})$ Bar graph depicts the prevalence of $\mathrm{S}$. aureus high samples at each skin site in psoriasis lesional (blue bars) and psoriasis non-lesional (orange bars) skin. (e) Bacterial species associated with $\mathrm{S}$. aureus high samples (red bars) and S. aureus low samples (green bars). Figure S4. Expression of Th1 components in effector T cells in response to Staphylococcus aureus colonization. The expression of Th1 components (a) T-bet (b) IFNy and (c)IL-2 are comparable in all experimental groups. Figure S5. Expression of Th2 components in effector T cells in response to Staphylococcus aureus colonization. The expression of Th2 cytokines (a) IL-4 (b) IL-5, (c) IL-13 (d) IL-9 are comparable in all experimental groups. The expression of Th2 promoting transcription factor (e) GATA3 is induced by early colonization of $\mathrm{S}$. aureus (adj. $p$-value $=1.49 \mathrm{e}-16$ ); whereas, another Th2 transcription factor ( $f$ STAT6 is not significantly induced. (PDF $405 \mathrm{~kb}$ )
\end{abstract}

Additional file 2: $\mathrm{R}$ notebook for applying linear mixed effects model to test the significance of difference in microbial diversity among psoriatic lesional, psoriatic unaffected, and healthy skin. (HTML $772 \mathrm{~kb}$ )

Additional file 3: Output of GraphPad Prism for statistics of heterogeneity of microbial communities isolated from skin associated with different disease state. (XML $18 \mathrm{~kb})$

Additional file 4: $\mathrm{R}$ notebook for microbe-microbe correlation analyses at genus and species level. (HTML $1518 \mathrm{~kb}$ )

Additional file 5: OTU table summarized to genus level used for correlation analysis. (CSV $1031 \mathrm{~kb}$ )

Additional file 6: OTU table summarized to species level used for correlation analysis. (CSV $295 \mathrm{~kb}$ )

Additional file 7: Mann-Kendall Test to detect trend in alpha diversity. (HTML $785 \mathrm{~kb})$

\section{Acknowledgements}

We thank the patients who generously provided samples for this study. This study was supported in part by a National Psoriasis Foundation Translation Research Award and NIH grants to WL (R01AR065174, U01Al119125) and National Psoriasis Foundation Fellowship Award to DY. TCS is supported by K08AR068409 and Burroughs Wellcome Fund CAMS-1015631. Flow cytometry was performed in the UCSF Parnassus Flow Cytometry Core which is supported by the Diabetes Research Center (DRC) grant, NIH P30 Dk063720. NGS sequencing was performed in Gladstone Genomics Core. We thank Mr. Kevin Lai for his computational assistance on this project.

\section{Availability of data and materials}

$16 \mathrm{~S}$ rRNA sequence data is deposited in the European Nucleotide Archive (http://www.ebi.ac.uk/ena) under study accession number PRJEB25915. RNAseq sequence data is deposited in the Gene Expression Omnibus (https://www.ncbi.nlm.nih.gov/geo/) under study accession number GSE114398. 


\section{Authors' contributions}

$\mathrm{HC}$ designed the 165 rRNA profiling experiment, performed the bioinformatics analyses, and wrote the manuscript. DY, RS, and KL recruited all participants and collected the demographic data from patients. HC and DY performed all DNA extraction and 165 rRNA library construction. DF helped design the statistical analyses. DY, RS, KL, XL, DU, and LA collected patient the skin swab samples. JL helped design the cutaneous microbiome profiling analyses. KV and JL performed the mouse Staphylococcus colonization experiment and provide RNAseq data. ML helped in the RNAseq analysis. HC, SV, MR, TS, and WL supervised the project. All authors reviewed and agreed upon the final manuscript.

\section{Competing interests}

The authors declare that they have no competing interests.

\section{Publisher's Note}

Springer Nature remains neutral with regard to jurisdictional claims in published maps and institutional affiliations.

\section{Author details}

'Department of Dermatology, University of California, San Francisco, CA 94115, USA. ${ }^{2}$ Cleveland Clinic Lerner College of Medicine, Cleveland, $\mathrm{OH}$ 44106, USA. ${ }^{3}$ University of Miami Miller School of Medicine, Miami, FL 33136, USA. ${ }^{4}$ Department of Internal Medicine, Yale University, New Haven, CT 06520, USA. 5Dermatology Department, Peking University Third Hospital, Beijing, China. ${ }^{6}$ Department of Dermatology, Dicle University School of Medicine, 21280 Diyarbakır, Turkey. ${ }^{7}$ Division of Gastroenterology, University of California, San Francisco, San Francisco, CA 94143, USA.

\section{Received: 10 April 2018 Accepted: 13 August 2018}

\section{Published online: 05 September 2018}

\section{References}

1. Roberson EDO, Bowcock AM. Psoriasis genetics: breaking the barrier. Trends Genet. 2010;26:415-23. Elsevier Ltd

2. Afifi L, Danesh MJ, Lee KM, Beroukhim K, Farahnik B, Ahn RS, et al. Dietary behaviors in psoriasis: patient-reported outcomes from a U.S. national survey. Dermatol Ther (Heidelb). 2017;7:227-42. Springer

3. Cai Y, Fleming C, Yan J. New insights of T cells in the pathogenesis of psoriasis. Cell Mol Immunol. 2012;9:302-9.

4. Grice EA, Segre JA. The skin microbiome. Nat Rev Microbiol. 2011;9:244-53

5. Belkaid Y, Tamoutounour S. The influence of skin microorganisms on cutaneous immunity. Nat Rev Immunol. 2016;16:353-66. Nature Publishing Group, a division of Macmillan Publishers Limited. All Rights Reserved

6. Naik S, Bouladoux N, Wilhelm C, Molloy MJ, Salcedo R, Kastenmuller W, et al. Compartmentalized control of skin immunity by resident commensals. Science. 2013:337:1115-9.

7. Ganju P, Nagpal S, Mohammed M, Nishal Kumar P, Pandey R, Natarajan VT, et al. Microbial community profiling shows dysbiosis in the lesional skin of vitiligo subjects. Sci Rep. 2016;6:18761.

8. Kong HH, Oh J, Deming C, Conlan S, Grice EA, Beatson MA, et al. Temporal shifts in the skin microbiome associated with disease flares and treatment in children with atopic dermatitis. Genome Res. 2012;22:850-9. Cold Spring Harbor Laboratory Press

9. Gudjonsson JE, Thorarinsson AM, Sigurgeirsson B, Kristinsson KG, Valdimarsson $\mathrm{H}$. Streptococcal throat infections and exacerbation of chronic plaque psoriasis: a prospective study. Br J Dermatol. 2003;149:530-4. Blackwell Science Ltd

10. Telfer NR, Chalmers RJG, Whale K, Colman G. The role of streptococcal infection in the initiation of guttate psoriasis. Arch Dermatol. 1992;128:39. American Medical Association

11. Lowes MA, Suárez-Fariñas M, Krueger JG. Immunology of psoriasis. Annu Rev Immunol. 2014;32:227-55. NIH Public Access

12. Alekseyenko AV, Perez-Perez Gl, De Souza A, Strober B, Gao Z, Bihan M, et al. Community differentiation of the cutaneous microbiota in psoriasis. Microbiome. 2013;1:31

13. Fahlén A, Engstrand L, Baker BS, Powles A, Fry L. Comparison of bacterial microbiota in skin biopsies from normal and psoriatic skin. Arch Dermatol Res. 2012;304:15-22.

14. Gao Z, Tseng C, Strober BE, Pei Z, Blaser MJ. Substantial alterations of the cutaneous bacterial biota in psoriatic lesions. PLoS One. 2008;3:e2719.
15. Tett A, Pasolli E, Farina S, Truong DT, Asnicar F, Zolfo M, et al. Unexplored diversity and strain-level structure of the skin microbiome associated with psoriasis. NPJ Biofilms Microbiomes. 2017;3:14.

16. Loesche MA, Farahi K, Capone K, Fakharzadeh S, Blauvelt A, Duffin KC, et al. Longitudinal study of the psoriasis-associated skin microbiome during therapy with ustekinumab in a randomized phase $3 b$ clinical trial. J Invest Dermatol. 2018; Elsevier. https://doi.org/10.1016/j.jid.2018.03.1501

17. Kong HH, Andersson B, Clavel T, Common JE, Jackson SA, Olson ND, et al. Performing skin microbiome research: a method to the madness. J Invest Dermatol. 2017;137:561-8. NIH Public Access

18. Jo J-H, Kennedy EA, Kong HH. Research techniques made simple: bacterial 165 ribosomal RNA gene sequencing in cutaneous research. J Invest Dermatol. 2016:136:e23-7. NIH Public Access

19. Cutting, Mary A, Mclnnes P. Manual of procedures for Human Microbiome Project core microbiome sampling Protocol A HMP Protocol \# 07-001 Version 12.0. NC.fB Inf. 2010;

20. Kong HH. Details matter: designing skin microbiome studies. J Invest Dermatol. 2016;136:900-2. NIH Public Access

21. Oh J. Human, Bacterial and Fungal Amplicon Collection and Processing for Sequencing. Bio-protocol. 2015;5(10): e1477. https://doi.org/10.21769/ BioProtoc.1477.

22. Meisel JS, Hannigan GD, Tyldsley AS, SanMiguel AJ, Hodkinson BP, Zheng Q, et al. Skin microbiome surveys are strongly influenced by experimental design. J Invest Dermatol. 2016;136:947-56. Elsevier Ltd

23. Costello EK, Lauber CL, Hamady M, Fierer N, Gordon Jl, Knight R. Bacterial community variation in human body habitats across space and time. Science. 2009;326:1694-7. NIH Public Access

24. Grice EA, Kong HH, Conlan S, Deming CB, Davis J, Young AC, et al. Topographical and temporal diversity of the human skin microbiome. Science. 2009:324:1190-2. NIH Public Access

25. Human Microbiome Project Consortium THMP. Structure, function and diversity of the healthy human microbiome. Nature. 2012;486:207-14. NIH Public Access

26. Oh J, Byrd AL, Park M, Kong HH, Segre JA. Temporal stability of the human skin microbiome. Cell. 2016:165:854-66.

27. Turnbaugh PJ, Hamady M, Yatsunenko T, Cantarel BL, Duncan A, Ley RE, et al. A core gut microbiome in obese and lean twins. Nature. 2009;457:480-4. Nature Publishing Group

28. Qin J, Li R, Raes J, Arumugam M, Burgdorf KS, Manichanh C, et al. A human gut microbial gene catalogue established by metagenomic sequencing. Nature. 2010;464:59-65. Nature Publishing Group

29. Simonis N, Rual J-FJ, Carvunis A-R, Tasan M, Lemmens I, Hirozane-kishikawa $T$, et al. Empirically controlled mapping of the Caenorhabditis elegans protein-protein interactome network. Nat Methods. 2008;6:47-54

30. Fredricks DN, Fiedler TL, Marrazzo JM. Molecular identification of bacteria associated with bacterial vaginosis. N Engl J Med. 2005;353:1899-911. Massachusetts Medical Society

31. Srinivasan S, Hoffman NG, Morgan MT, Matsen FA, Fiedler TL, Hall RW, et al. Bacterial communities in women with bacterial vaginosis: high resolution phylogenetic analyses reveal relationships of microbiota to clinical criteria. PLoS One. 2012;7:e37818. Ratner AJ, editor. Public Libr Sci

32. Lozupone C, Knight R. UniFrac: a new phylogenetic method for comparing microbial communities. Appl Environ Microbiol. 2005;71:8228-35. American Society for Microbiology (ASM)

33. Segata N, Izard J, Waldron L, Gevers D, Miropolsky L, Garrett WS, et al. Metagenomic biomarker discovery and explanation. Genome Biol. 2011;12: R60. BioMed Central

34. Otto M, Echner H, Voelter W, Götz F. Pheromone cross-inhibition between Staphylococcus aureus and Staphylococcus epidermidis. Infect Immun. 2001;69:1957-60. American Society for Microbiology

35. Perez Perez Gl, Gao Z, Jourdain R, Ramirez J, Gany F, Clavaud C, et al. Body site is a more determinant factor than human population diversity in the healthy skin microbiome. PLoS One. 2016;11:e0151990. Public Libr Sci

36. McHeyzer-Williams M, Okitsu S, Wang N, McHeyzer-Williams L. Molecular programming of B cell memory. Nat Rev Immunol. 2011;12:24-34. NIH Public Access

37. Bröker BM, Mrochen D, Péton V. The T cell response to Staphylococcus aureus. Pathog (Basel, Switzerland). 2016;5 Multidisciplinary Digital Publishing Institute (MDPI)

38. Kolata JB, Kühbandner I, Link C, Normann N, Vu CH, Steil L, et al. The fall of a dogma? Unexpected high T-cell memory response to Staphylococcus aureus in humans. J Infect Dis. 2015;212:830-8. Oxford University Press 
39. Ishigame H, Kakuta S, Nagai T, Kadoki M, Nambu A, Komiyama Y, et al. Differential roles of interleukin-17A and -17F in host defense against mucoepithelial bacterial infection and allergic responses. Immunity. 2009;30: 108-19.

40. Byrd AL, Deming C, Cassidy SKB, Harrison OJ, Ng W-I, Conlan S, et al. Staphylococcus aureus and Staphylococcus epidermidis strain diversity underlying pediatric atopic dermatitis. Sci Transl Med. 2017;9:eaal4651. American Association for the Advancement of Science

41. Brandt EB, Sivaprasad U. Th2 cytokines and atopic dermatitis. J Clin Cell Immunol. 2011;2:110. NIH Public Access

42. Nestle FO, Kaplan DH, Barker J. Psoriasis. N Engl J Med. 2009;361:496-509.

43. Knight R, Vrbanac A, Taylor BC, Aksenov A, Callewaert C, Debelius J, et al. Best practices for analysing microbiomes. Nat Rev Microbiol. 2018;1 Nature Publishing Group

44. Totté JEE, van der Feltz WT, Bode LGM, van Belkum A, van Zuuren EJ, Pasmans SGMA. A systematic review and meta-analysis on Staphylococcus aureus carriage in psoriasis, acne and rosacea. Eur J Clin Microbiol Infect Dis. 2016;35:1069-77. Springer

45. Leyden JJ, Marples RR, Kligman AM. Staphylococcus aureus in the lesions of atopic dermatitis. Br J Dermatol. 1974;90:525. Blackwell Publishing Ltd

46. Oyoshi MK, Murphy GF, Geha RS. Filaggrin-deficient mice exhibit TH17dominated skin inflammation and permissiveness to epicutaneous sensitization with protein antigen. J Allergy Clin Immunol. 2009;124:485-93, 493.e1. NIH Public Access

47. Bojar RA, Holland KT. Acne and propionibacterium acnes. Clin Dermatol. 2004;22:375-9.

48. Leeming JP, Holland KT, Cuncliffe WJ. The microbial colonization of inflamed acne vulgaris lesions. Br J Dermatol. 1988;118:203-8. Blackwell Publishing Ltd

49. Tomida S, Nguyen L, Chiu B-H, Liu J, Sodergren E, Weinstock GM, et al. Pan-genome and comparative genome analyses of propionibacterium acnes reveal its genomic diversity in the healthy and diseased human skin microbiome. MBio. 2013;4:e00003-13. American Society for Microbiology (ASM)

50. Fitz-Gibbon S, Tomida S, Chiu B-H, Nguyen L, Du C, Liu M, et al. Propionibacterium acnes strain populations in the human skin microbiome associated with acne. J Invest Dermatol. 2013;133:2152-60. NIH Public Access

51. Barnard E, Shi B, Kang D, Craft N, Li H. The balance of metagenomic elements shapes the skin microbiome in acne and health. Sci Rep. 2016;6: 39491. Nat Publ Group

52. Smith PM, Howitt MR, Panikov N, Michaud M, Gallini CA, Bohlooly-Y M, et al The microbial metabolites, short-chain fatty acids, regulate colonic Treg cell homeostasis. Science. 2013;341:569-73. NIH Public Access

53. Allhorn M, Arve S, Brüggemann H, Lood R. A novel enzyme with antioxidant capacity produced by the ubiquitous skin colonizer Propionibacterium acnes. Sci Rep. 2016;6:36412. Nat Publ Group

54. Agak GW, Kao S, Ouyang K, Qin M, Moon D, Butt A, et al. Phenotype and Antimicrobial Activity of Th17 Cells Induced by Propionibacterium acnes Strains Associated with Healthy and Acne Skin. J Invest Dermatol. Elsevier; 2018;138:316-24.

55. Kloos WE. Natural populations of the genus Staphylococcus. Annu Rev Microbiol. 1980;34:559-92. Annual Reviews 4139 El Camino Way, P.O. Box 10139, Palo Alto, CA 94303-0139, USA

56. Hauschild T, Schwarz S. Differentiation of Staphylococcus sciuri strains isolated from free-living rodents and insectivores. J Vet Med Ser B. 2003;50: 241-6. Wiley/Blackwell (10.1111)

57. Couto I, Sanches IS, Sá-Leão R, de Lencastre H. Molecular characterization of Staphylococcus sciuri strains isolated from humans. J Clin Microbiol. 2000; 38:1136-43. American Society for Microbiology (ASM)

58. Bansal S, Jain A, Agarwal J. Prevalence of methicillin-resistant, coagulasenegative staphylococci in neonatal intensive care units: findings from a tertiary care hospital in India. J Med Microbiol. 2004;53:941-4.

59. Lang S, Livesley MA, Lambert PA, Elliott J, Elliott TT. The genomic diversity of coagulase-negative Staphylococci associated with nosocomial infections. J Hosp Infect. 1999;43:187-93. WB Saunders

60. Petinaki E, Kontos F, Miriagou V, Maniati M, Hatzi F, Maniatis A. Survey of methicillin-resistant coagulase-negative staphylococci in the hospitals of central Greece. Int J Antimicrob Agents. 2001;18:563-6. Elsevier

61. Ahoyo TA, Yehouenou Pazou E, Baba-Moussa L, Attolou Gbohou A, Boco M, Dramane $\mathrm{KL}$, et al. Staphylococcus sciuri outbreak at tertiary hospital in Benin. J Med Microbiol Diagnosis. 2013;2 OMICS International
62. de Sousa MA, Santos Sanches I, Ferro ML, de Lencastre H. Epidemiological study of Staphylococcal colonization and cross-infection in two West African Hospitals. Microb Drug Resist. 2000;6:133-41. Mary Ann Liebert, Inc

63. Dakić I, Morrison D, Vuković D, Savić B, Shittu A, Jezek P, et al. Isolation and molecular characterization of Staphylococcus sciuri in the hospital environment. J Clin Microbiol. 2005:43:2782-5. American Society for Microbiology (ASM)

64. Magoč T, Salzberg SL. FLASH: fast length adjustment of short reads to improve genome assemblies. Bioinformatics. 2011;27:2957-63. Oxford University Press

65. Caporaso JG, Kuczynski J, Stombaugh J, Bittinger K, Bushman FD, Costello EK, et al. QIIME allows analysis of high-throughput community sequencing data. Nat Methods. 2010;7:335-6.

66. Edgar RC. Search and clustering orders of magnitude faster than BLAST. Bioinformatics. 2010;26:2460-1. Oxford University Press

67. Caporaso JG, Bittinger K, Bushman FD, DeSantis TZ, Andersen GL, Knight R. PyNAST: a flexible tool for aligning sequences to a template alignment. Bioinformatics. 2010;26:266-7. Oxford University Press

68. Haas BJ, Gevers D, Earl AM, Feldgarden M, Ward DV, Giannoukos G, et al. Chimeric 16S rRNA sequence formation and detection in Sanger and 454pyrosequenced PCR amplicons. Genome Res. 2011;21:494-504. Cold Spring Harbor Laboratory Press

69. Price MN, Dehal PS, Arkin AP. FastTree 2 - approximately maximumlikelihood trees for large alignments. PLoS One. 2010;5:e9490. Poon AFY, editor. Public Libr Sci

70. Dobin A, Davis CA, Schlesinger F, Drenkow J, Zaleski C, Jha S, et al. STAR: ultrafast universal RNA-seq aligner. Bioinformatics. 2013;29:15-21. Oxford University Press

71. Li H, Handsaker B, Wysoker A, Fennell T, Ruan J, Homer N, et al. The sequence alignment/map format and SAMtools. Bioinformatics. 2009;25: 2078-9. Oxford University Press

72. Anders S, Pyl PT, Huber W. HTSeq--a Python framework to work with highthroughput sequencing data. Bioinformatics. 2015;31:166-9. Oxford University Press

73. Love MI, Huber W, Anders S. Moderated estimation of fold change and dispersion for RNA-seq data with DESeq2. Genome Biol. 2014;15:550. BioMed Central

74. Trapnell C, Roberts A, Goff L, Pertea G, Kim D, Kelley DR, et al. Differential gene and transcript expression analysis of RNA-seg experiments with TopHat and Cufflinks. Nat Protoc. 2012;7:562-78. Nature Publishing Group

\section{Ready to submit your research? Choose BMC and benefit from}

- fast, convenient online submission

- thorough peer review by experienced researchers in your field

- rapid publication on acceptance

- support for research data, including large and complex data types

- gold Open Access which fosters wider collaboration and increased citations

- maximum visibility for your research: over $100 \mathrm{M}$ website views per year

At $\mathrm{BMC}$, research is always in progress.

Learn more biomedcentral.com/submissions 